\title{
An RRx-001 Analogue With Potent Anti-NLRP3 Inflammasome Activity but Without High-Energy Nitro Functional Groups
}

\author{
Hualong Lin ${ }^{1,2,3}$, Mingyang Yang ${ }^{4}$, Cong $L^{1,2,3}$, Bolong Lin ${ }^{1,2,3}$, Xianming Deng ${ }^{4 *}$, \\ Hongbin $\mathrm{He}^{1,2,3 *}$ and Rongbin Zhou ${ }^{1,2,3 *}$
}

${ }^{1}$ Department of Geriatrics, First Affiliated Hospital of USTC, Division of Life Sciences and Medicine, University of Science and Technology of China, Hefei, China, ${ }^{2}$ CAS Key Laboratory of Innate Immunity and Chronic Disease, School of Basic Medical Sciences, Division of Life Sciences and Medicine, University of Science and Technology of China, Hefei, China, ${ }^{3}$ Chinese Academy of Sciences Centre for Excellence in Cell and Molecular Biology, University of Science and Technology of China, Hefei, China, ${ }^{4}$ State Key Laboratory of Cellular Stress Biology, Innovation Center for Cell Signaling Network, School of Life Sciences, Xiamen University, Xiamen, China

OPEN ACCESS

Edited by:

Tharmarajan Ramprasath,

Georgia State University,

United States

Reviewed by:

Rosalinda Sorrentino,

University of Salerno, Italy

Venkata Naga Lakshmi Ramarao Sure,

Tulane University, United States

*Correspondence:

Xianming Deng

xmdeng@xmu.edu.cn

Hongbin $\mathrm{He}$

hhb123@ustc.edu.cn

Rongbin Zhou

zrb1980@ustc.edu.cn

Specialty section:

This article was submitted to Inflammation Pharmacology,

a section of the journal

Frontiers in Pharmacology

Received: 26 November 2021

Accepted: 18 January 2022

Published: 17 February 2022

Citation:

Lin H, Yang $M, L i C$, Lin B, Deng $X$, He $H$ and Zhou R (2022) An RRx-001

Analogue With Potent Anti-NLRP3

Inflammasome Activity but Without

High-Energy Nitro Functional Groups.

Front. Pharmacol. 13:822833.

doi: 10.3389/fphar.2022.822833
NLRP3 inflammasome is involved in the pathology of multiple human inflammatory diseases but there are still no clinically available medications targeting the NLRP3 inflammasome. We have previously identified RRx-001 as a highly selective and potent NLRP3 inhibitor, however, it contains high-energy nitro functional groups and may cause potential processing problems and generates highly toxic oxidants. Here, we show that compound 149-01, an RRx-001 analogue without high-energy nitro functional groups, is a potent, specific and covalent NLRP3 inhibitor. Mechanistically, 149-01 binds directly to cysteine 409 of NLRP3 to block the NEK7-NLRP3 interaction, thereby preventing NLRP3 inflammasome complex assembly and activation. Furthermore, treatment with 149-01 effectively alleviate the severity of several inflammatory diseases in mice, including lipopolysaccharide (LPS)-induced systemic inflammation, monosodium urate crystals (MSU)-induced peritonitis and experimental autoimmune encephalomyelitis (EAE). Thus, our results indicate that $149-01$ is a potential lead for developing therapeutic agent for NLRP3-related inflammatory diseases.

Keywords: RRx-001 analogues, NLRP3 inflammasome, anti-inflammation, NLRP3-related inflammatory diseases, NLRP3 inhibitor

\section{INTRODUCTION}

The NLRP3 inflammasome is a multimeric protein complex consisted of innate immune sensor NLRP3 (NLR family, Pyrin domain containing 3), adaptor protein ASC and effector cysteine protease caspase-1, it plays a pivotal role in host defense against microbial infection and inflammation (Schroder and Tschopp, 2010; Davis et al., 2011). Unlike other sensor proteins, NLRP3 can be activated by a wide range of factors derived from microbes, the host and the environment (Broz and Dixit, 2016). Upon activation, NLRP3 recruits ASC, which then interacts with pro-caspase-1 to induce NLRP3 inflammasome complex assembly. This results in the cleavage and activation of caspase- 1 , which subsequently promotes the maturation and secretion of IL- $1 \beta$ and IL-18 and induces pyroptosis, a type of inflammatory cell death (Shi et al., 2015; Swanson et al., 2019). However, aberrant activation of NLRP3 inflammasome has been reported to promote the 
development of several human diseases, including gout, type 2 diabetes (T2D), inflammatory bowel disease (IBD), atherosclerosis, neurodegenerative diseases and others (Martinon et al., 2006; Bauer et al., 2010; Duewell et al., 2010; Masters et al., 2010; Wen et al., 2012; Heneka et al., 2013; Guo et al., 2015). Therefore, NLRP3 inflammasome is considered as a novel therapeutic target for these inflammatory diseases.

Currently, biologic agents targeting IL- $1 \beta$ are clinically used to treat NLRP3-driven diseases, including canakinumab (a neutralizing IL-1 $\beta$ monoclonal antibody), anakinra (a recombinant non-glycosylated IL-1 receptor antagonist), and rilonacept (a soluble decoy IL-1 $\beta$ receptor) (Dinarello et al., 2012). These agents have been proven to be effective in the treatment of Cryopyrin-associated periodic syndromes (CAPS) caused by NLRP3 gene mutations and have also been applied in clinical trials for other NLRP3-associated disorders (Dinarello et al., 2012; Dinarello and van der Meer, 2013). However, the NLRP3 inflammasome activation induces not only IL- $1 \beta$ production, but also drives pyroptosis and other proinflammatory cytokines production such as IL-18, which may also contribute to the pathogenesis of inflammatory diseases (Nowarski et al., 2015). Moreover, IL-1 $\beta$ can also be produced by other inflammasomes or in an inflammasomeindependent manner (Davis et al., 2011; Netea et al., 2015), so blockage of IL- $1 \beta$ may lead to a higher risk of infection. Therefore, compared with biologic agents that target IL-1 $\beta$, inhibitors that directly target NLRP3 inflammasome may be a better therapeutic option for NLRP3-related diseases.

In recent years, several small molecules, including MCC950, CY-09, OLT1177, oridonin, tranilast, INF39 and others, have been proposed to inhibit the NLRP3 inflammasome activation and exert remarkable therapeutic effects for NLRP3-associated disorders in animal models (Coll et al., 2015; Daniels et al., 2016; Jiang et al., 2017; He et al., 2018; Huang et al., 2018; Marchetti et al., 2018; Zhang et al., 2020; Shi et al., 2021). However, considering the safety, efficacy and specificity of these inhibitors, only a few have entered clinical trials, such as the MCC950-related agents (Inzomelid and IZD334), IFM-2427, OLT1177 (Mullard, 2019; Kluck et al., 2020). Although these agents hold promise for the treatment of NLRP3-related diseases, their clinical efficacy and safety remain to be further determined.

We previously described RRx-001 (1-bromoacetyl-3,3dinitroazetidine), an antitumor agent in phase III clinical trials, as a novel potent NLRP3 inhibitor (Kim et al., 2016; Oronsky et al., 2019; Chen et al., 2021). However, RRx-001 is a small-molecule compound developed from the defense and aerospace industry and contains unique high-energy nitro functional groups (Oronsky et al., 2016). The gemdinitroazetidine can decompose rapidly and may be sensitive to heat, impact, friction and electrostatic discharge (Ning et al., 2012; Straessler et al., 2012). Due to its inherent energy properties, the gem-dinitroazetidine can cause potential processing problems. In addition, these geminal dinitro-groups undergo decomposition under hypoxic conditions in vivo, producing the gaseous multifunctional free radical, nitric oxide (NO) (Fens et al., 2011; Oronsky et al., 2017). Further chemical interaction between $\mathrm{NO}$ and ROS (reactive oxygen species) can generate highly toxic oxidants, including nitrogen dioxide and peroxynitrite (van der Vliet et al., 2000; Oronsky et al., 2017). Moreover, our previous study has shown that removal of geminal dinitro-groups from $\mathrm{RRx}-001$ resulted in a nearly 32-fold decrease in its inhibitory activity (Chen et al., 2021).

In this study, we tried to identify $R R x-001$ analogues with potent anti-NLRP3 inflammasome activity but without highenergy nitro functional groups. Our results showed that 14901 , an RRx-001 analogue, had comparable activity with RRx-001. Mechanistically, 149-01 blocked the NEK7-NLRP3 interaction by binding directly to cysteine 409 of NLRP3, thereby preventing the assembly of NLRP3 inflammasome complex. Moreover, 149-01 treatment effectively attenuated several NLRP3-related inflammatory diseases in mice, including LPS-induced systemic inflammation, MSU-induced peritonitis and EAE. Taken together, our findings demonstrate that $149-01$ is active both in vitro and in vivo, suggesting that 149-01 is a potential lead for developing therapeutic agent for NLRP3-related human inflammatory diseases.

\section{MATERIALS AND METHODS}

\section{Mice}

C57BL/6J mice were obtained from Shanghai SLAC Laboratory Animal Limited Liability Company (Shanghai, China). The generation of $\mathrm{Nlrp3}^{-/-}$mice were as previously described (Martinon et al., 2006). Mice were maintained in a SPF facility under a 12/12 h light/dark cycle (lights on at 07:00 and off at 19: $00)$. All animal studies were performed in accordance with the guidelines of the Ethics Committee of University of Science and Technology of China.

\section{Reagents}

ATP, Nigericin, MSU, and poly (A/T) were purchased from Sigma-Aldrich. Ultrapure LPS, Lipofextamine 2000, Pam3CSK4, MQAE, MitoSOX, MitoTracker, DAPI were acquired from Invitrogen. The Salmonella strain was a gift from Dr. Cai Zhang (Shandong University, Shandong, China). The C3 toxin was a gift from Dr. Tengchuan Jin (University of Science and Technology of China, Hefei, China). CY-09 was synthesized from Dr. Xianming Deng (Xiamen University, Xiamen, China). Protein G agarose (16-266) and anti-Flag (A2220) beads were bought from Millipore and SigmaAldrich, respectively. Anti-Flag (F2555) and anti-VSV (V4888) antibodies were from Sigma-Aldrich. The anti-mouse NLRP3 (AG-20B-0014) and the anti-mouse caspase-1 (AG-20B-0042) antibodies was from AdipoGen. The anti-mouse IL- $1 \beta$ antibody (AF-401-NA) was obtained from R\&D Systems. The anti- $\beta$-actin antibody (66009-1-Ig) was from Proteintech Group. The antimouse ASC antibody (67824S) was from Cell Signaling Technology. The anti-mouse NEK7 antibody (ab133514) was from Abcam.

\section{Cell Preparation and Stimulation}

BMDMs (bone marrow-derived macrophages) were collected from the bone marrow of 6-8 weeks old mice and cultured in 
DMEM supplemented with 10\% FBS and $20 \mathrm{ng} / \mathrm{ml}$ murine M-CSF (Novoprotein). Human PBMCs (peripheral blood mononuclear cells) were obtained by Human Lymphocyte Separation Medium (catalog no. P8610-200, Solarbio) and cultured in RPMI 1640 medium containing 10\% FBS.

To stimulate inflammasome activation, BMDMs $\left(5 \times 10^{5} /\right.$ well $)$ or PBMCs $\left(1 \times 10^{6} /\right.$ well $)$ were seeded into 12 -well plates and cultured overnight. Cells were primed for $3 \mathrm{~h}$ in Opti-MEM with LPS $(50 \mathrm{ng} / \mathrm{ml})$ or Pam3CSK $4 \quad(400 \mathrm{ng} / \mathrm{ml}$, for noncanonical inflammasome activation), followed by treatment with 149-01 for $30 \mathrm{~min}$, and then stimulated with various inflammasome agonists: $3 \mu \mathrm{M}$ nigericin $(30 \mathrm{~min}) ; 150 \mu \mathrm{g} / \mathrm{ml}$ MSU (4h); $2.5 \mathrm{mM}$ ATP (30 min); S. typhimurium (4 h, multiplicity of infection (MOI)); $30 \mu \mathrm{M}$ CL097 ( $1 \mathrm{~h})$ or $0.5 \mu \mathrm{g} /$ $\mathrm{ml} \mathrm{C} 3$ toxin $(6 \mathrm{~h}) .0 .5 \mu \mathrm{g} / \mathrm{ml}$ poly $(\mathrm{A} / \mathrm{T})$ or $500 \mathrm{ng} / \mathrm{ml} \mathrm{LPS} \mathrm{were}$ transfected into BMDMs with Lipofectamine 2000 for $4 \mathrm{~h}$ or $16 \mathrm{~h}$, respectively. The release of lactate dehydrogenase (LDH) was measured by LDH Cytotoxicity Assay Kit (Beyotime). Precipitated supernatants and cell lysates were further detected using western blotting.

In vitro, 149-01 was dissolved in DMSO, and the DMSO was used as vehicle control in all figures.

\section{ELISA}

Cell culture supernatants and serum samples were analyzed for mouse IL-6, TNF- $\alpha$ or IL- $1 \beta$ and for human TNF- $\alpha$ or IL- $1 \beta$ with ELISA kit (all from R\&D except human IL-1 $\beta$ from BD, 557953) according to the manufacturer's instructions.

\section{Western Blotting}

Sample buffer was added to resuspend precipitated supernatants or to lyse cells, and the cell lysates were transferred to $1.5 \mathrm{ml} \mathrm{EP}$ tube. All protein samples were further boiled at $100^{\circ} \mathrm{C}$ for $10 \mathrm{~min}$ and separated by $8 \%$ or $15 \%$ SDS-PAGE gels at $80 \mathrm{~V}$ for $0.5 \mathrm{~h}$ and at $120 \mathrm{~V}$ for $1 \mathrm{~h}$. After that, proteins were transferred to PVDF membranes at $90 \mathrm{~V}$ for $1 \mathrm{~h}$. The membranes were blocked with PBST containing 5\% nonfat milk at room temperature for $1 \mathrm{~h}$ and then incubated with primary and conjugated secondary antibodies as described previously (Chen et al., 2021). After washing the membranes with PBST, protein bands were acquired after visualization by enhanced chemiluminescence.

\section{Detection of Intracellular $\mathrm{K}^{+}$and $\mathrm{Cl}^{-}$Levels}

To determine intracellular $\mathrm{K}^{+}$concentration, BMDMs $\left(1 \times 10^{6} /\right.$ well) seeded in 6-well plates were stimulated with nigericin to trigger NLRP3 inflammasome activation as described above. After that, cell culture supernatants were thoroughly removed and cells were lysed with ultrapure $\mathrm{HNO}_{3}$. Cell lysates were further boiled at $100^{\circ} \mathrm{C}$ for $30 \mathrm{~min}$ and then the precipitated products were dissolved with $\mathrm{ddH}_{2} \mathrm{O} . \mathrm{K}^{+}$concentrations were measured using PerkinElmer Optima 2000 DV spectrometer.

To determine intracellular $\mathrm{Cl}^{-}$concentration, BMDMs seeded in 12-well plates were also stimulated to activate NLRP3 inflammasome. After stimulation, the culture supernatants were thoroughly discarded and cells were lysed with $\mathrm{ddH}_{2} \mathrm{O}$ $(200 \mu \mathrm{l} /$ well $)$ at $37^{\circ} \mathrm{C}$ for $30 \mathrm{~min}$. Cell lysates were transferred to $1.5 \mathrm{ml} \mathrm{EP}$ tube, centrifuged at $10,000 \times \mathrm{g}$ for $5 \mathrm{~min}$ and then supernatants were mixed with MQAE $(10 \mu \mathrm{M})$. The absorbance of the mixture was determined using BioTek Multi-Mode Microplate Readers (Synergy2). A control was set for each experiment to measure the extracellular residual $\mathrm{Cl}^{-}$level after removal of the culture supernatant.

\section{Confocal Microscopy}

BMDMs $\left(2 \times 10^{5} /\right.$ well) were seeded on coverslips (Thermo Fisher Scientific) and cultured overnight in 12 -well plates. The next day, LPS-primed mouse BMDMs were stained with $5 \mu \mathrm{M}$ MitoSOX or $50 \mathrm{nM}$ MitoTracker Red and stimulated with nigericin. After stimulation, the culture supernatants were removed, and the cells were fixed in situ with 4\% paraformaldehyde (PFA) in PBS for 15 min after washing by ice-cold PBS three times. Then, the cells were washed three times with PBST. Confocal microscopic analyses were utilized to detect mitochondrial damage by using a Zeiss LSM 700.

\section{Immunoprecipitation}

To detect endogenous interaction, LPS-primed BMDMs were stimulated with nigericin in 6-well plates, then lysed in NP-40 lysis buffer containing protease inhibitor cocktail. The cell lysates were centrifuged at $10,000 \mathrm{rpm}$ at $4^{\circ} \mathrm{C}$ for $10 \mathrm{~min}$ and the supernatants were then incubated with primary antibodies and Protein G Mag Sepharose overnight with rotation at $4^{\circ} \mathrm{C}$. The protein-antibody complex was pulled down by protein $\mathrm{G}$ beads and determined using immunoblot analysis.

To detect exogenous interaction, plasmids were transfected into HEK-293T cells in 6-well plates via polyethylenimine (PEI). $24 \mathrm{~h}$ after transfection, cells were collected and lysed in NP-40 lysis buffer. After centrifugation, supernatants of cell lysates were co-incubated with anti-Flag antibody-coated beads. Then, immunocomplex proteins were pulled down by beads and analyzed using immunoblotting.

\section{ASC Oligomerization Assay}

BMDMs $\left(1 \times 10^{6} /\right.$ well $)$ were plated in 6 -well plates and treated with nigericin. After stimulation, the cells were washed with ice-cold PBS and then lysed in NP-40 lysis buffer at $4^{\circ} \mathrm{C}$ for $30 \mathrm{~min}$. The cell lysates were centrifuged at $330 \times \mathrm{g}$ at $4^{\circ} \mathrm{C}$ for $10 \mathrm{~min}$. The pellets were washed with ice-cold PBS three times and re-suspended in $500 \mu \mathrm{l}$ PBS, then cross-linked with $2 \mathrm{mM}$ disuccinimidyl suberate (DSS, Sangon Biotech, C100015) at room temperature for $30 \mathrm{~min}$ with rotation. The crosslinked pellets were collected after centrifugation at $330 \mathrm{~g}$ for $10 \mathrm{~min}$ and dissolved directly in $30 \mu \mathrm{l}$ sample buffer, and analyzed using immunoblotting.

\section{Protein Expression and Purification}

To purify human Flag-NLRP3 protein, human Flag-NLRP3 plasmid was transfected into HEK-293T cells via PEI and expressed for $48 \mathrm{~h}$. The cells were then washed with ice-cold PBS and lysed in lysis buffer (50 mM HEPES, pH 7.4, $150 \mathrm{mM}$ $\mathrm{NaCl}$, and $0.4 \% \mathrm{CHAPS}$ ) at $4^{\circ} \mathrm{C}$ for $30 \mathrm{~min}$. The cell lysates were centrifuged at $14,000 \mathrm{rpm}$ at $4^{\circ} \mathrm{C}$ for $15 \mathrm{~min}$. After centrifugation, supernatants of cell lysates were coincubated with anti-Flag antibody-coated beads at $4^{\circ} \mathrm{C}$ for 
$2.5 \mathrm{~h}$ with rotation. The beads were collected after centrifugation and incubated with Flag peptide (SigmaAldrich, F3290) in elution buffer (50 mM HEPES, pH 7.4, $500 \mathrm{mM} \mathrm{NaCl}$ and $0.1 \%$ CHAPS) at $4^{\circ} \mathrm{C}$ for $90 \mathrm{~min}$ with rotation to elute Flag-NLRP3 protein. The eluents containing Flag-NLRP3 protein were collected and concentrated by ultrafiltration device (UFC910024; Merck Millipore) to remove proteins $<100 \mathrm{kD}$. Meanwhile, $10 \mu \mathrm{l}$ eluent was dissolved in sample buffer, separated by $8 \%$ SDS-PAGE gels, followed by detection using anti-Flag antibody.

To purify human GFP-NLRP3 protein, human His-GFPNLRP3 plasmid was transfected into HEK-293T cells via PEI and expressed for $48 \mathrm{~h}$. The cells were lysed in lysis buffer at $4^{\circ} \mathrm{C}$ for $30 \mathrm{~min}$ and sonicated. The supernatants of cell lysates were then collected by centrifuging at $14,000 \mathrm{rpm}$ at $4^{\circ} \mathrm{C}$ for $15 \mathrm{~min}$. The His-GFP-NLRP3 proteins were preliminarily isolated using nickel-nitrilotriacetic acid matrices (QIAGEN) at room temperature for $45 \mathrm{~min}$, and the pulled down proteins were washed twice with lysis buffer. After that, proteins were eluted in elution buffer and the eluents were concentrated by ultrafiltration device to remove proteins $<10 \mathrm{kD}$ after filtration through a $0.45 \mu \mathrm{m}$ syringe filter. Then, the final eluents were added to a Superdex 200 10/ $300 \mathrm{GL}$ column (GE Healthcare) for further purification in $50 \mathrm{mM}$ HEPES ( $\mathrm{pH} 7.4$ ) and $150 \mathrm{mM} \mathrm{NaCl}$ on an GE Healthcare AKTA purifier. The solutions containing HisGFP-NLRP3 protein were collected together and concentrated by ultrafiltration device to remove proteins $<100 \mathrm{kD}$. Meanwhile, $10 \mu \mathrm{l}$ solution was dissolved in sample buffer and analyzed using immunoblotting.

\section{NLRP3 ATPase Activity Assay}

Purified human NLRP3 protein $(1.4 \mathrm{ng} / \mu \mathrm{l})$ was incubated with indicated doses of $149-01$ or $1 \mu \mathrm{M} \mathrm{CY}-09$ in reaction buffer at $37^{\circ} \mathrm{C}$ for $15 \mathrm{~min}$ and then ATP $(25 \mu \mathrm{m}$, Ultra-Pure ATP $)$ was added. After further incubation at $37^{\circ} \mathrm{C}$ for $40 \mathrm{~min}$, the amount of ATP converted into ADP in the mixture was measured by luminescent ADP detection using ADP-Glo Kinase Assay kit (Promega, Madison, MI, United States). The data were presented as percentage of residual enzyme activity versus vehicle-treated enzyme activity.

\section{Microscale Thermophoresis Assay}

A Monolith NT.115 instrument (NanoTemper Technologies) was used to measure the $K_{\mathrm{D}}$ value. Purified His-GFP-NLRP3 protein $(200 \mathrm{nM})$ was incubated at room temperature with a range of doses of $149-01$ (from $1 \mu \mathrm{M}$ to $0.0169 \mathrm{nM}$ ) in assay buffer (50 mM HEPES, $10 \mathrm{mM} \mathrm{MgCl}_{2}, 100 \mathrm{mM} \mathrm{NaCl}, \mathrm{pH} 7.5$, and $0.05 \%$ Tween 20 ) for $40 \mathrm{~min}$. After the mixtures were loaded onto the NanoTemper glass capillaries, measurements were performed using $80 \%$ MST power and $100 \%$ LED power. $K_{\mathrm{D}}$ value calculations were done using the mass action equation through NanoTemper software from duplicate reads of an experiment.

\section{Drug Affinity Responsive Target Stability Assays}

Assays were performed according to published protocols. BMDMs primed with LPS ( $3 \mathrm{~h}$ ) or HEK-293T cells transfected with plasmid $(24 \mathrm{~h})$ were collected and lysed in NP-40 lysis buffer containing protease inhibitor cocktail. The cell lysates were centrifuged at $12,000 \times g$ at $4^{\circ} \mathrm{C}$ for $10 \mathrm{~min}$ and the supernatants were then measured by Pierce BCA Protein Assay Kit (Beyotime) to determine the protein concentration. After that, lysates $(8 \mu \mathrm{g}$ of protein lysate per reaction) were incubated at $4^{\circ} \mathrm{C}$ with indicated doses of 149-01 overnight with rotation. The mixtures were further incubated with protease pronase ( $25 \mathrm{ng}$ of enzyme per $\mu \mathrm{g}$ of protein, Sigma) at room temperature for another $30 \mathrm{~min}$. Then, the samples were dissolved directly in sample buffer and analyzed using immunoblotting.

\section{NLRP3 Reconstitution}

$\mathrm{Nlrp} 3^{-1-}$ BMDMs were seeded into 12-well plates and cultured overnight. Then, cells were transduced with lentivirus (pLEX vector, Thermo Fisher) encoding mouse NLRP3 or their indicated mutant $(\mathrm{C} 405 \mathrm{~A})$. After $6 \mathrm{~h}$, the culture supernatant was removed and replaced with DMEM containing 10\% FBS and $20 \mathrm{ng} / \mathrm{ml}$ murine M-CSF. After $48 \mathrm{~h}, \mathrm{BMDMs}$ were stimulated with nigericin.

\section{Histological Analysis}

Mouse spinal cords were fixed in $4 \%$ PFA at $4^{\circ} \mathrm{C}$ for $24 \mathrm{~h}$, embedded in paraffin, and sectioned. Then, sections were stained with H\&E or LFB according to standard procedures. Histological analysis for inflammatory cell infiltration and demyelination was performed with a Nikon ECL IPSE Ci biological microscope, and images were recorded using a Nikon DS-U3 color digital camera.

\section{Quantitative Real-Time PCR}

Total RNA from tissues was extracted using TRIzol reagent (Takara). To synthesize cDNA, $800 \mathrm{ng}$ of total RNA from each sample was reverse transcribed with Moloney murine leukemia virus (M-MLV) reverse transcriptase (Invitrogen). Then qPCR analysis was performed with SYBR Green premix (Takara Bio). All data were individually normalized to Gapdh. The primer sequences are shown as follows:

for mouse Gapdh: forward, GGTGAAGGTCGGTGTGAA CG; reverse, CTCGCTCCTGGAAGATGGTG; for mouse Illb: forward, TGCCACCTTTTGACAGTGATG; reverse, AAGGTC CACGGGAAAGACAC; for mouse Tnf: forward, CGATGGGTT GTACCTTGTC; reverse, CGGACTCCGCAAAGTCTAAG; for mouse Il6: forward, GTCCTTCCTACCCCAATTTCC; reverse, GCACTAGGTTTGCCGAGTAGA.

\section{LPS-Induced Systemic Inflammation}

8-week-old male mice were injected intraperitoneally with $5 \mathrm{mg} / \mathrm{kg} 149-01$ or vehicle control (90\% PBS plus 10\% DMSO) for $30 \mathrm{~min}$ and then challenged with $20 \mathrm{mg} / \mathrm{kg}$ LPS. After $4 \mathrm{~h}$, 
mice were sacrificed and the secretion of IL- $1 \beta$ and TNF- $\alpha$ in serum was detected by ELISA.

\section{MSU-Induced Peritonitis}

8 -week-old male mice pretreated with $5 \mathrm{mg} / \mathrm{kg} 149-01$ or vehicle (90\% PBS plus 10\% DMSO) for 30 min were intraperitoneally injected with $1 \mathrm{mg}$ MSU (dissolved in $0.5 \mathrm{ml} \mathrm{PBS}$ ). After $6 \mathrm{~h}$, mice were sacrificed and peritoneal cavities were washed with $10 \mathrm{ml}$ ice-cold PBS. The proportion of polymorph nuclear neutrophils was detected by flow cytometry and the number of peritoneal exudate cells was counted. In addition, the secretion of IL-1 $\beta$ in lavage fluid or serum was also measured using ELISA.

\section{Induction and Assessment of EAE}

8 -week-old male mice were subcutaneously immunized with $300 \mu \mathrm{g} \mathrm{MOG}_{35-55}$ peptide emulsified in CFA supplemented with $5 \mathrm{mg} / \mathrm{ml}(0.5 \mathrm{mg} / \mathrm{mouse})$ of heat-killed Mycobacterium tuberculosis. Mice were intravenously injected with $150 \mathrm{ng}$ pertussis toxin on days 0 and $2.149-01 \quad(5 \mathrm{mg} / \mathrm{kg})$ was administered intraperitoneally to mice on the day of induction and every 2 days thereafter. Control mice were given vehicle $(90 \%$ PBS plus $10 \%$ DMSO) at the same time points. Clinical disease scores were recorded according to the following standard scale: 0 , no abnormalities; 1 , limp tail or waddling gait with tail tonicity; 2 , wobbly gait; 3 , hind limb paralysis; 4 , hind limb and forelimb paralysis; 5, death. Mononuclear cells isolated from both the brain and spinal cord using 30\% Percoll separation were analyzed by flow cytometry.

\section{Statistical Analyses}

All data are presented as mean \pm s.e.m. Statistical analysis was carried out with one-way ANOVA (GraphPad Software) for data in all figures (except Figure 7 and Supplementary Figure S7) or with unpaired Student's t-tests (GraphPad Software) for data in Figure 7 and Supplementary Figure S7, and differences were considered significant at $p<.05$. No values were excluded.

\section{RESULTS}

\section{9-01 Blocks NLRP3 Inflammasome Activation}

Several RRx-001 analogues were screened for their NLRP3 inhibitory activity in Supplementary Figure S1A and their chemical structure were depicted in Supplementary Table S1. In the screening results of RRx-001 analogues, 149-01 showed comparable NLRP3 inhibitory activity with RRx-001. To further evaluate the inhibitory effect of 149-01 on NLRP3 inflammasome activation, mouse bone marrow-derived macrophages (BMDMs) were first primed with LPS, then pretreated with 149-01 for $30 \mathrm{~min}$, lastly stimulated with the NLRP3 stimulus nigericin. We found that 149-01 dose-dependently inhibited IL-1 $\beta$ release and caspase-1 activation at the doses of 200-600 nM (Figures 1A-C). Consistently, 149-01 also suppressed nigericin-induced release of lactate dehydrogenase (LDH) (Figure 1D), but LPS-dependent IL-6 and TNF- $\alpha$ secretion was not affected (Figures 1E,F), which suggests that 149-01 inhibits NLRP3-dependent cytokine production and pyroptosis. To ascertain whether 149-01 only blocked NLRP3 activation induced by nigericin, we tested the effects of 149-01 on NLRP3 activation triggered by two additional agonists, ATP and MSU. We observed that 149-01 inhibited IL-1 $\beta$ release and caspase-1 activation triggered by both NLRP3 agonists (Figures 1G,H). In addition, 149-01 also dose-dependently suppressed intracellular LPS-induced noncanonical NLRP3 inflammasome activation (Supplementary Figures S2A,B) but did not reduce LDH release (Supplementary Figure S2C). As previously reported, intracellular LPS induced $\mathrm{LDH}$ release was independent of NLRP3 (Coll et al., 2015). These results demonstrate that 149-01 displays potent and broad inhibitory effects on both canonical and non-canonical NLRP3 activation.

We next investigated whether 149-01 was effective for human cells. We isolated human peripheral blood mononuclear cells (PBMCs), treated LPS-primed cells with 149-01 for $30 \mathrm{~min}$ and then activated NLRP3 inflammasome with nigericin. We observed that $149-01$ treatment reduced IL- $1 \beta$ release in a dose-dependent manner but not TNF- $\alpha$ production (Figures 1I,J). Furthermore, LPS alone induced alternative NLRP3 inflammasome activation was also suppressed by 149-01 in PBMCs (Supplementary Figures S2D,E). These results together indicate that 149-01 can prevent NLRP3 inflammasome activation in both mouse and human cells.

Because $\mathrm{RRx}-001$ is reported to inhibit pro-IL- $1 \beta$ expression in macrophages (Yu et al., 2019; Chen et al., 2021), we then tested whether 149-01 influenced LPS-induced priming phase of NLRP3 inflammasome activation. We found that at concentrations of 200-600 nM, 149-01 treatment before or after LPS stimulation did not affect LPS-induced pro-IL-1 $\beta$, NLRP3 production and IL6 , TNF- $\alpha$ secretion (Supplementary Figures S3A-C), indicating that 149-01 does not influence LPS-induced priming phase at concentrations that blocks NLRP3 activation. Although at higher concentrations of 1-4 $\mu \mathrm{M}, 149-01$ treatment before LPS stimulation markedly inhibited LPS-induced pro-IL- $1 \beta$ and IL6 production but did not affect NLRP3 or TNF- $\alpha$ expression (Supplementary Figures S4A-C), 149-01 showed approximately 9 times inhibitory activity against IL- $1 \beta$ release than that against IL-6 production (Supplementary Figures S4D,E). Besides, higher concentrations (one to four $\mu \mathrm{M}$ ) of 149-01 treatment after LPS stimulation did not affect LPS-induced pro-IL-1 $\beta$, NLRP3 production and IL-6, TNF-a secretion (Supplementary Figures S4A-C).

\section{9-01 Does Not Affect AIM2, NLRC4, or Pyrin Inflammasomes Activation}

Besides NLRP3, several other sensors were also proposed to trigger inflammasome complex assembly (Xu et al., 2014; Broz and Dixit, 2016). To explore the specificity of 149-01's inhibitory activity against NLRP3 inflammasome, we examined whether 149-01 could suppress the activation of three other wellcharacterized inflammasome. Murine BMDMs were first primed with LPS, then transfected with double-stranded DNA analog poly (A/T) to induce the AIM2 (Absent In Melanoma 2) inflammasome activation or infected with $\mathrm{S}$. typhimurium to 
A<smiles>O=C(CBr)NCc1ccc(C(F)(F)F)cc1</smiles>

149-01
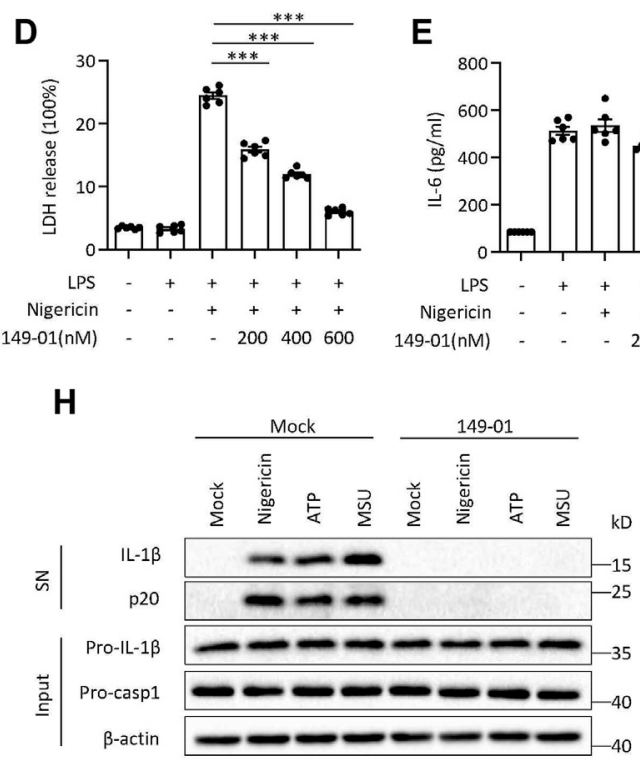

E
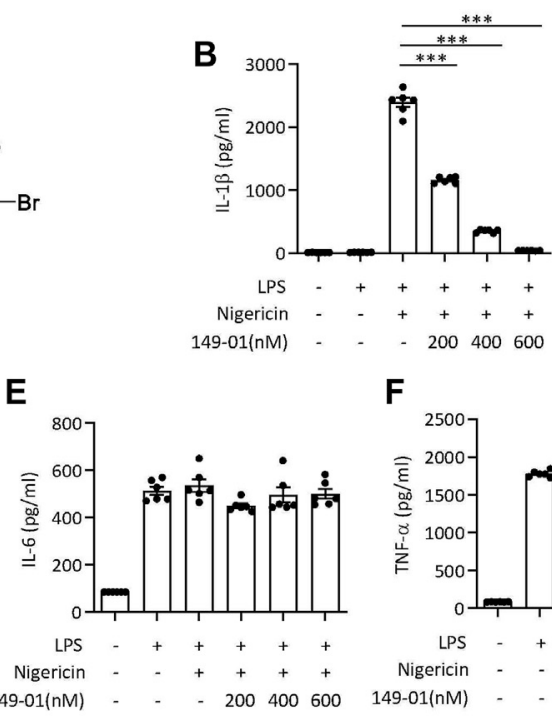

$\mathbf{F}$

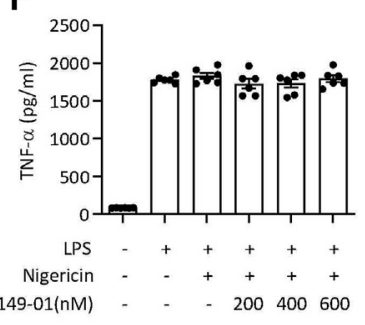

I

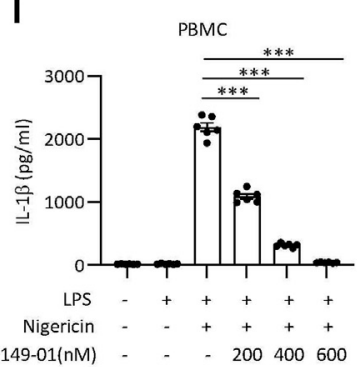

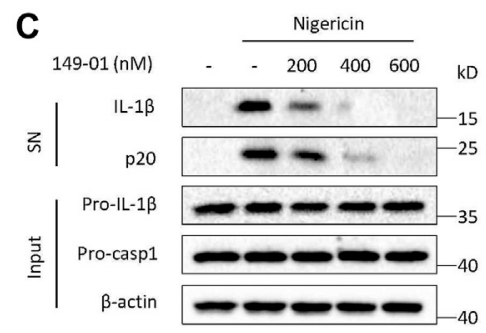

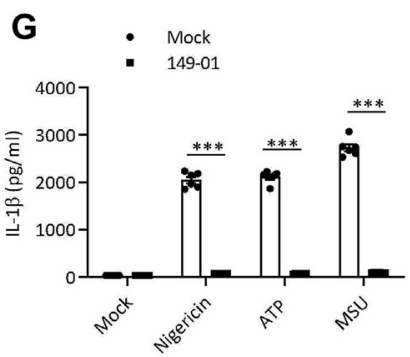

J

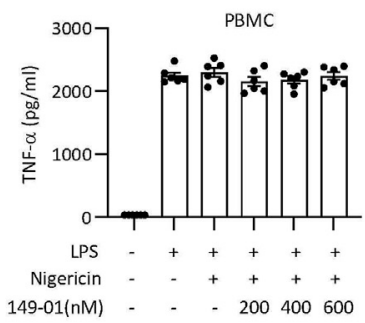

FIGURE 1 | Identification of 149-01 as a highly potent inhibitor for NLRP3 inflammasome. (A) 149-01 structure. (B-F) BMDMs were first primed with LPS for $3 \mathrm{~h}$, then pretreated with indicated doses of 149-01 (200-600 nM) for $30 \mathrm{~min}$, lastly stimulated with nigericin. (B) IL-1 $\beta$ releases in supernatants were detected by ELISA. (C) Active IL-1 $\beta$ and p20 (cleaved caspase-1) in supernatants (SN) and pro-IL-1 $\beta$, pro-caspase-1 and $\beta$-actin in cell lysates (Input) were measured by western blot. (D) The release of LDH in supernatants. (E) IL-6 and (F) TNF- $\alpha$ secretion levels in supernatants were determined by ELISA. (G,H) BMDMs were first primed with LPS, then treated with or without 149-01 (600 nM) for 30 min, lastly stimulated with nigericin, ATP or MSU. (G) IL-1 $\beta$ releases in supernatants were detected by ELISA. (H) Active $\mathrm{IL}-1 \beta$ and $\mathrm{p} 20$ in supernatants and pro-IL-1 $\beta$, pro-caspase-1 and $\beta$-actin in cell lysates were measured by western blot. (I,J) PBMCs were first primed with LPS for $3 \mathrm{~h}$, then pretreated with indicated doses of 149-01 for 30 min, lastly stimulated with nigericin. (I) IL-1 $\beta$ and (J) TNF- $\alpha$ secretion levels in supernatants were determined by ELISA. Data are obtained from three independent experiments, each with two biological replicates and are expressed as mean \pm S. e.m $(n=6)(\mathbf{B , D}-\mathbf{G}, \mathbf{I}, \mathbf{J})$, or are representative of three independent experiments $\mathbf{( C , H )}$. One-way ANOVA was applied to calculate statistical significance: ${ }^{* \star *} p<.001$.

induce the NLRC4 (NLR Family CARD Domain Containing 4) inflammasome activation or stimulated with $\mathrm{C} 3$ toxin to induce the Pyrin inflammasome activation. In contrast to its potent inhibitory effects on NLRP3 inflammasome activation, 149-01 could not inhibit IL-1 $\beta$ release or caspase- 1 activation following AIM2 or NLRC4 inflammasome activation (Figures 2A,B). In addition, 149-01 also could not attenuate IL-1 $\beta$ release triggered by Pyrin inflammasome activation (Figure 2C). Collectively, our data show that 149-01 selectively suppresses NLRP3 inflammasome activation.

\section{9-01 Suppresses the Assembly of NLRP3 Inflammasome by Preventing the NEK7-NLRP3 Interaction}

Next, we sought to identify the precise mechanism by which 149 01 prevents NLRP3 inflammasome activation. Potassium $\left(\mathrm{K}^{+}\right)$ efflux and chloride $\left(\mathrm{Cl}^{-}\right)$efflux are proposed to be upstream events required for NLRP3 activation (Munoz-Planillo et al., 2013; Tang et al., 2017). To determine whether 149-01 abrogates NLRP3 activation by affecting these upstream events, we tested intracellular levels of $\mathrm{K}^{+}$and $\mathrm{Cl}^{-}$after nigericin stimulation. We found that pretreatment with 149-01 in BMDMs did not prevent the nigericin-induced decrease in intracellular $\mathrm{K}^{+}$and $\mathrm{Cl}^{-}$(Supplementary Figures S5A,B). Consistent with this, we also observed that 149-01 could dosedependently suppress NLRP3 inflammasome activation induced by CL097, which is a $\mathrm{K}^{+}$efflux-independent NLRP3 agonist (Supplementary Figure S5C) (Gross et al., 2016). In addition, mitochondrial damage is considered to be another upstream event required for NLRP3 activation (Zhou et al., 2011; Gurung et al., 2015). We next detected the mitochondrial status of BMDMs after nigericin stimulation and observed abnormal mitochondrial fission, clustering, and enhanced ROS production. However, pretreatment with 149-01 did not significantly affect these processes (Supplementary Figure S5D). Taken together, our 

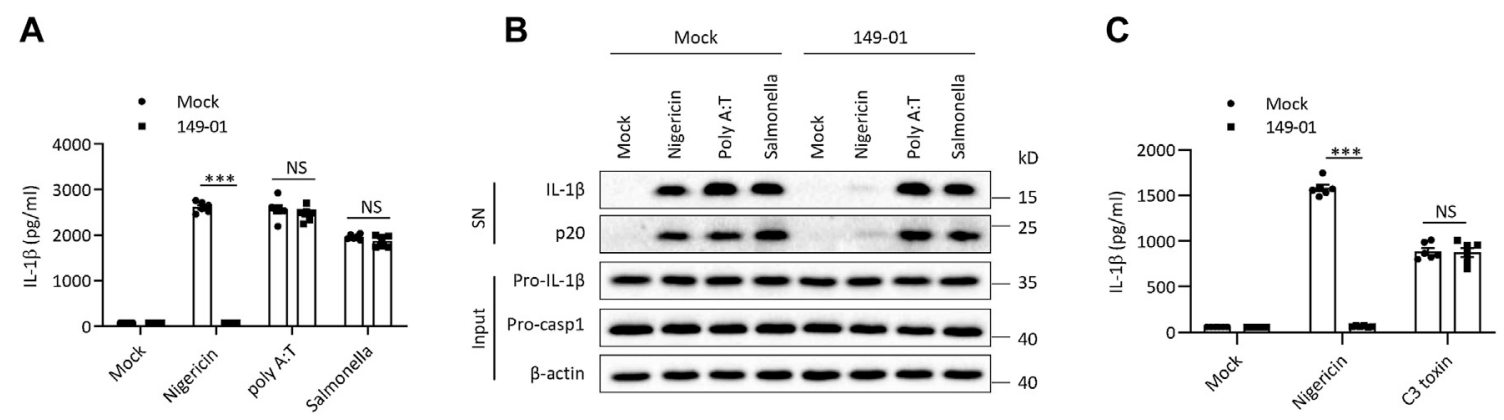

FIGURE 2 | 149-01 does not affect the activation of AIM2, NLRC4, or Pyrin inflammasomes. (A-C) BMDMs were first primed with LPS, then treated with or without 149-01 (600 nM) for 30 min, lastly stimulated with nigericin, poly (AVT), Salmonella or C3 toxin. (A,C) IL-1 $\beta$ releases in supernatants were detected by ELISA. (B) Active IL$1 \beta$ and p20 in supernatants and pro-IL-1 $\beta$, pro-caspase- 1 and $\beta$-actin in cell lysates were measured by western blot. Data are obtained from three independent experiments, each with two biological replicates and are expressed as mean \pm s. e.m $(n=6)(\mathbf{A}, \mathbf{C})$, or are representative of three independent experiments $(\mathbf{B})$. Oneway ANOVA was applied to calculate statistical significance: ${ }^{\star \star \star} p<.001, \mathrm{NS}$, not significant.

A

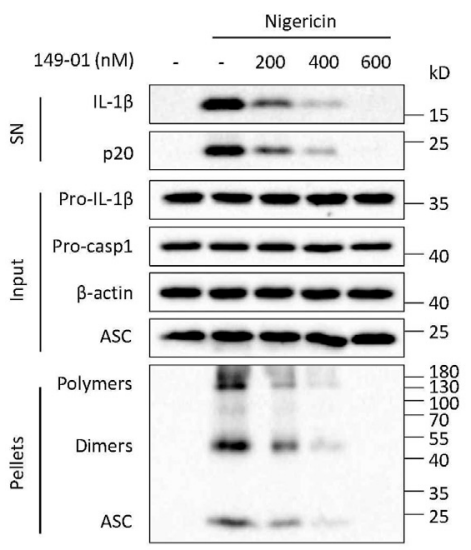

B

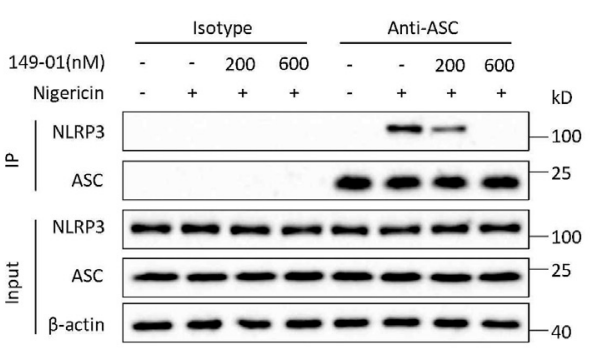

C

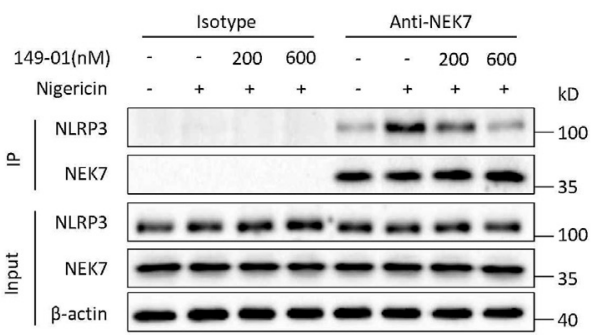

D

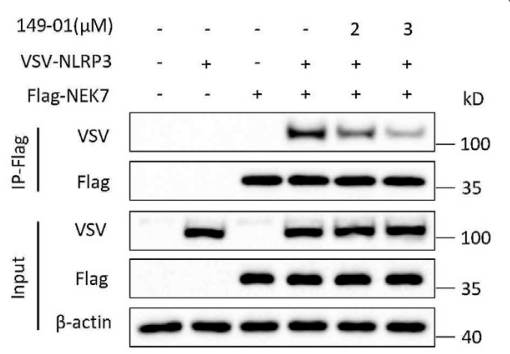

E

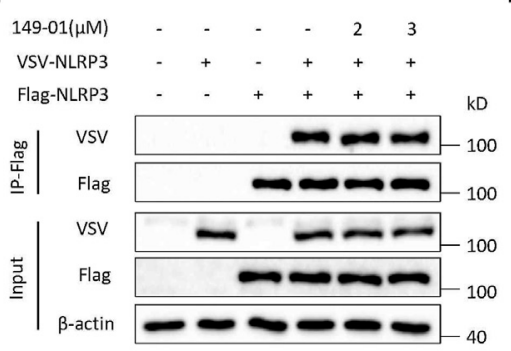

$\mathbf{F}$

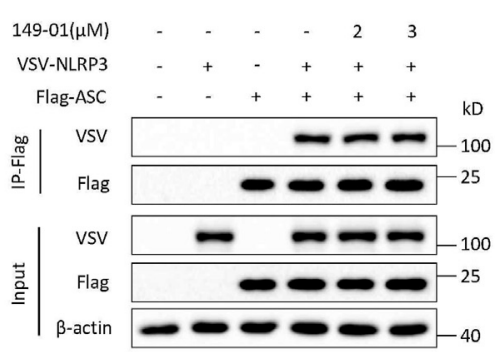

FIGURE 3 | 149-01 blocks the assembly of NLRP3 inflammasome by preventing the interaction between NEK7 and NLRP3. (A) Supernatants, cell lysates and DSS-crosslinked pellets from LPS-primed BMDMs treated with indicated doses of 149-01 for 30 min before nigericin stimulation were analyzed by western blot. (B) The endogenous NLRP3-ASC interaction in LPS-primed BMDMs treated with indicated doses of 149-01 for 30 min before nigericin stimulation was detected by immunoprecipitation (IP) and western blot. (C) The endogenous NEK7-NLRP3 interaction in LPS-primed BMDMs treated with indicated doses of 149-01 for $30 \mathrm{~min}$ before nigericin stimulation was detected by IP and western blot. (D) The NEK7-NLRP3 interaction in HEK-293T cells transfected with Flag-NEK7, VSV-NLRP3 plasmids and treated with indicated doses of 149-01 was evaluated by IP and western blot. (E) The NLRP3-NLRP3 interaction in HEK-293T cells transfected with FlagNLRP3, VSV-NLRP3 plasmids and treated with indicated doses of 149-01 was evaluated by IP and western blot. (F) The NLRP3-ASC interaction in HEK-293T cells transfected with Flag-ASC, VSV-NLRP3 plasmids and treated with indicated doses of 149-01 was evaluated by IP and western blot. Data are representative of three independent experiments. 
findings indicate that 149-01 blocks NLRP3 activation by acting downstream of $\mathrm{K}^{+}$efflux, $\mathrm{Cl}^{-}$efflux and mitochondrial damage.

We then tested whether 149-01 could block NLRP3 activation by influencing NLRP3 inflammasome complex assembly. A critical step for NLRP3 inflammasome complex assembly and subsequent caspase-1 activation is ASC oligomerization (Lu et al., 2014; Dick et al., 2016), which can be detected after chemical cross-linking followed by western blotting. Consistent with inhibition of IL-1 $\beta$ release and caspase-1 activation, 149-01 markedly suppressed ASC oligomerization induced by nigericin at the doses of $200-600 \mathrm{nM}$ without affecting ASC expression in cell lysates (Figure 3A), indicating that 149-01 prevents NLRP3 activation by acting upstream of ASC oligomerization. Next, we examined by coimmunoprecipitation whether 149-01 could block NLRP3-ASC interaction, which is essential for ASC recruitment and subsequent ASC oligomerization (Swanson et al., 2019). We observed that 14901 dramatically attenuated the interaction between endogenous NLRP3 and ASC in nigericin stimulated BMDMs (Figure 3B). Moreover, we investigated if 149-01 influenced NEK7-NLRP3 interaction, which is imperative for NLRP3 oligomerization and subsequent NLRP3-ASC complex formation (He et al., 2016; Shi et al., 2016). We found that 149-01 substantially abolished the nigericin-mediated endogenous NEK7-NLRP3 interaction (Figure 3C). Collectively, these findings reveal that 149-01 may prevents NLRP3 inflammasome complex assembly by blocking the NEK7-NLRP3 interaction.

To validate this hypothesis, we overexpressed Flag-NEK7 and VSV-NLRP3 in HEK-293T cells, and explored by coimmunoprecipitation whether 149-01 could inhibit direct NEK7-NLRP3 interaction. The results showed that 149-01 treatment blocked the direct NEK7-NLRP3 interaction (Figure 3D). In contrast, 149-01 treatment had no impact on the direct NLRP3-NLRP3 or NLRP3-ASC interaction (Figures 3E,F). Therefore, these results suggest that 149-01 inhibits NLRP3 inflammasome activation by abrogating the direct NEK7-NLRP3 interaction, rather than by affecting NLRP3 oligomerization or subsequent NLRP3-ASC complex formation. Consistent with this, we found that 149-01 did not impact the NLRP3 ATPase activity (Supplementary Figure S6A), which is critical for the NLRP3 self-oligomerization (Duncan et al., 2007). In the same experiment, the known NLRP3 inflammasome inhibitor CY-09 was used as a positive control (Jiang et al., 2017). In addition, NEK7 has also been reported to act downstream of NEK9 and contribute to mitotic progression (Haq et al., 2015). However, 149-01 treatment did not affect the interaction between NEK7 and NEK9 (Supplementary Figure S6B).

\section{9-01 Binds Directly to Cys409 of NLRP3}

We next determined whether the activation of NLRP3 inflammasome inhibited by 149-01 was reversible. LPS-primed mouse BMDMs were first treated with 149-01 for $15 \mathrm{~min}$, then washed every $5 \mathrm{~min}$ for three times to remove unbound 149-01, and lastly stimulated with nigericin. We observed that nigericininduced IL-1 $\beta$ release remained inhibited after removal of unbound 149-01, suggesting that 149-01's inhibitory effect on NLRP3 activation is essentially irreversible (Figure 4A). In contrast, the inhibitory effects of CY-09 on NLRP3 activation is reversible (Supplementary Figure S6C). Together these data suggest that 149-01 may bind its target protein in a covalent manner.

Based on the result that 149-01 blocks the direct NEK7NLRP3 interaction, we inferred that 149-01 may prevents NLRP3 inflammasome complex assembly by directly targeting NEK7 or NLRP3. To confirm this conjecture, we performed the drug affinity responsive target stability (DARTS) assay (Lomenick et al., 2009; Coll et al., 2019), which exploits the target protein's reduced sensitivity to protease when it interacts with small molecules. We incubated the LPS-primed BMDMs cell lysates with different concentrations of 149-01, then digested with the protease pronase, and observed that 149-01 dose-dependently reduced proteolysis of NLRP3 by pronase (Figure 4B), suggesting that 149-01 directly interacts with NLRP3. However, incubation with 149-01 did not affect pronase mediated degradation of other NLRP3 inflammasome components, including NEK7, pro-casp1 and ASC (Figure 4B), indicating that 149-01 specifically targets NLRP3. To further validate the direct binding of 149-01 to NLRP3, we employed microscale thermophoresis (MST) assay to quantify the direct interaction between 149-01 and purified GFP-NLRP3. The data revealed that 149-01 interacted with purified GFP-NLRP3 with an equilibrium dissociation constant $\left(\mathrm{K}_{\mathrm{D}}\right)$ of $48.2 \mathrm{nM}$ (Figure $4 \mathrm{C}$ ). Therefore, our results reveal that 149-01 binds directly to NLRP3 with high affinity. To investigate whether 149-01 interacted with other inflammasome sensors, we overexpressed flag-tagged NLRP3, NLRP1b, AIM2 or NLRC4 in HEK-293T cells and then performed DARTS experiments. The results showed that 149-01 protected only NLRP3 but not NLRP1b, AIM2 or NLRC4 from pronaseinduced proteolysis in a dose-dependent manner (Figures 4D-G).

We next overexpressed three functional domains of NLRP3 in HEK-293T cells, NACHT, LRR and PYD, respectively, to explore which domain is responsible for interacting with 149-01. We found that only the NACHT domain but not the LRR or PYD domain could be protected by 149-01 from pronase-mediated proteolysis (Figures $\mathbf{5 A - C}$ ). These results together demonstrate that 149-01 directly and specifically targets the NACHT domain of NLRP3. Next, we sought to determine the 149-01-binding residue within the NLRP3 NACHT domain. Since RRx-001 has been reported to bind to cysteine 409 (Chen et al., 2021), we tested whether 149-01 also targeted the C409 site of the NACHT domain. Flag-tagged the NACHT domain with the C409A mutation was overexpressed in HEK-293T cells and then performed DARTS assay. We observed that the C409A mutation abrogated the interaction between 149-01 and the NACHT domain (Figure 5D). Furthermore, the C409A mutation had no effect on the NEK7-NLRP3 interaction, but abolished 149-01's inhibitory effect on this interaction (Figure 5E), suggesting that 149-01 binds directly to C409 of NLRP3. We then reconstituted $N L R P 3^{-/-}$BMDMs with mouse WT NLRP3 or mutant NLRP3 (C405A, corresponding to human NLRP3-C409A) and found that nigericin-induced NLRP3 
A

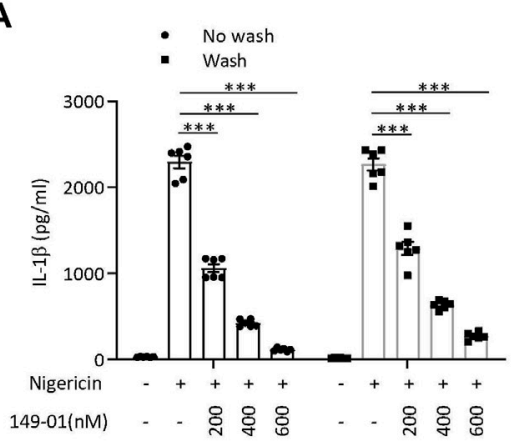

B

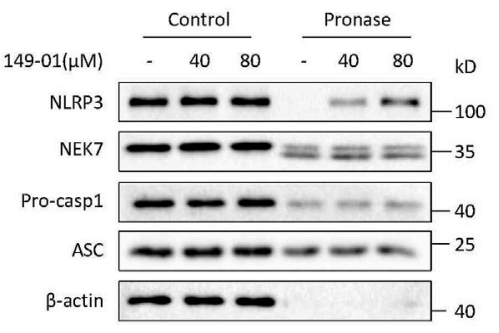

C

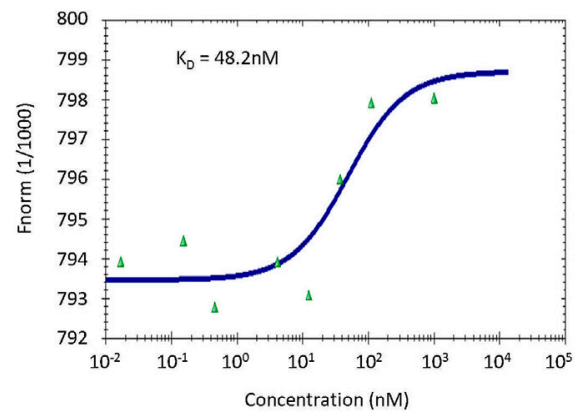

D

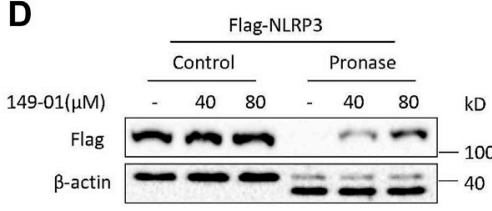

E

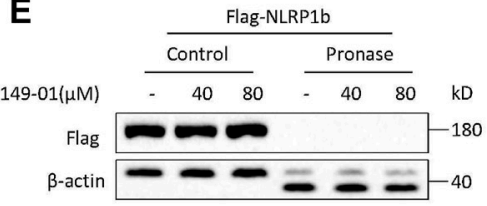

$\mathbf{F}$

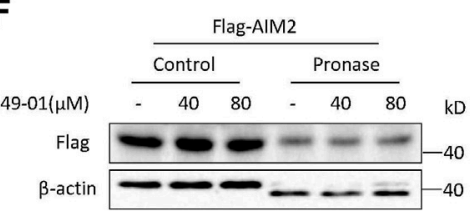

G

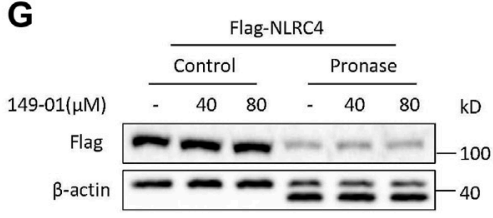

FIGURE 4 | 149-01 irreversibly, directly, and specifically binds to NLRP3. (A) IL-1 $\beta$ releases in supernatants from LPS-primed BMDMs treated with indicated doses of 149-01 for 15 min, washed three times before nigericin stimulation were detected by ELISA. (B) LPS-primed BMDMs cell lysates incubated overnight with indicated doses of 149-01 before pronase digestion were detected by western blot using antibodies for NLRP3, NEK7, pro-caspase-1, ASC and $\beta$-actin. (C) Binding affinity of 149-01 to purified GFP-NLRP3 protein was analyzed by MST. HEK-293T cells transfected with Flag-tagged NLRP3 (D), NLRP1b (E), AIM2 (F), or NLRC4 (G) were lysed and the cell lysates incubated overnight with indicated doses of 149-01 before pronase digestion were detected by western blot. Data are obtained from three independent experiments, each with two biological replicates and are expressed as mean \pm s. e.m $(n=6)(\mathbf{A})$, or are representative of three independent experiments (B-G). One-way ANOVA was applied to calculate statistical significance: ${ }^{\star \star \star} p<.001$.

activation was blocked by 149-01 in cells reconstituted with WT NLRP3 but remained intact in cells reconstituted with mutant NLRP3 (Figures 5F,G). Collectively, our results suggest that 14901 binds directly to cysteine 409 of NLRP 3 and then prevents the NEK7-NLRP3 interaction and ultimately inhibits NLRP3 inflammasome activation.

\section{9-01 Suppresses NLRP3 Inflammasome in vivo}

Since 149-01 disrupts NLRP3 inflammasome activation in vitro, we next tested the therapeutic efficacy of 149-01 in NLRP3-dependent septic shock disease model, in which intraperitoneal injection of LPS could elicit NLRP3-driven IL-1 $\beta$ production (He et al., 2013). Mice were injected with 149-01 intraperitoneally before challenge with LPS and were evaluated $4 \mathrm{~h}$ later. The data revealed that pretreatment with 149-01 markedly downregulated serum IL-1 $\beta$ level without considerably decreasing NLRP3-independent TNF- $\alpha$ production in serum (Figures 6A,B). These findings indicate that 149-01 suppresses LPS-induced NLRP3-related systemic inflammation in vivo.
MSU crystal-induced peritonitis is another well-established NLRP3-dependent acute inflammatory model characterized by IL- $1 \beta$ secretion and massive neutrophil infiltration in peritoneal cavity (Martinon et al., 2006). To investigate the inhibitory activity of 149-01 in peritonitis model, mice were pretreated with 149-01 before intraperitoneal injection of MSU. As expected, pretreatment with 149-01 efficiently inhibited IL-1 $\beta$ secretion and neutrophil recruitment (Figures 6C-E). Our results suggest that 149-01 significantly alleviates MSU-induced peritonitis in mice.

The experimental autoimmune encephalomyelitis (EAE) mouse model, which effectively simulates human multiple sclerosis disease, is characterized by inflammation and demyelination. Considering that NLRP3 inflammasome acts a key role in the pathogenesis of EAE and exacerbates neuroinflammation (Sutton et al., 2006; Gris et al., 2010; Lalor et al., 2011), we then assessed whether 149-01 could alleviate the severity of EAE via targeting NLRP3 inflammasome. We induced EAE by subcutaneous immunizing mice with myelin oligodendrocyte glycoprotein (MOG) peptide $\left(\mathrm{MOG}_{35-55}\right)$ and recorded clinical disease scores on a daily basis. Compared with the vehicle-treated group, treatment of mice with 149-01 
A

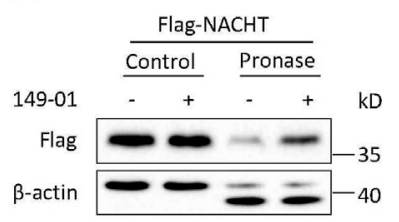

C

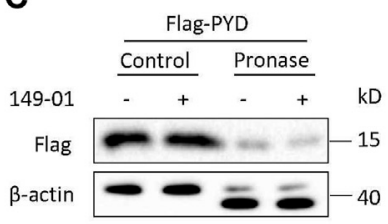

B

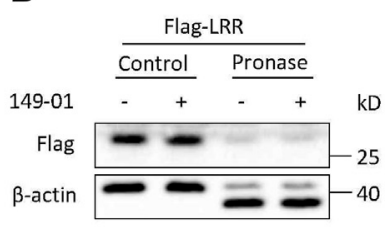

D

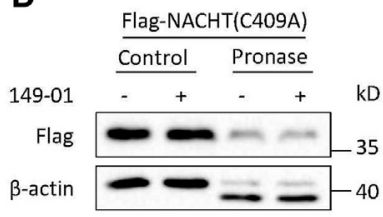

E

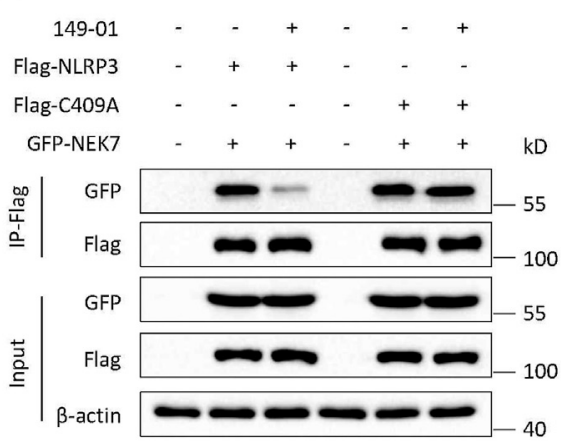

F

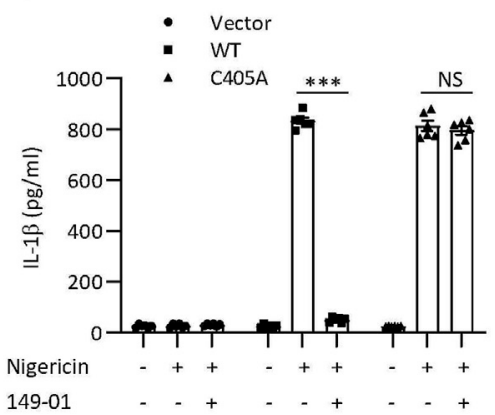

G

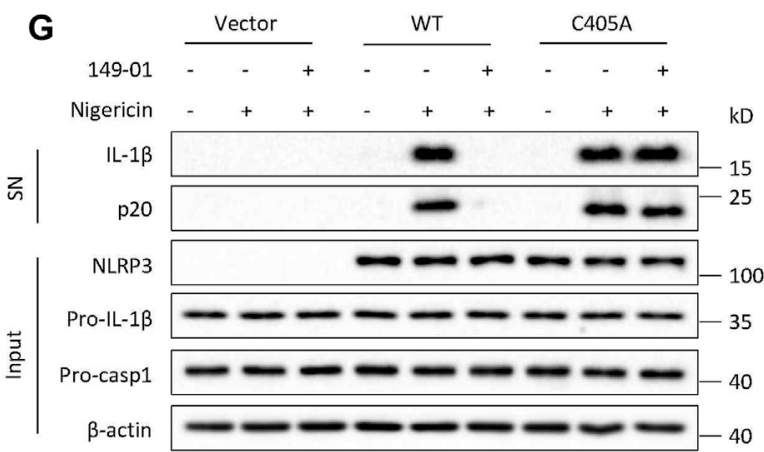

FIGURE 5 | 149-01 targets Cys409 of NLRP3. HEK-293T cells transfected with Flag-tagged NACHT (A), LRR (B), PYD (C), or NACHT (C409A) (D) were lysed and the cell lysates incubated overnight with or without 149-01 (80 $\mu \mathrm{M})$ before pronase digestion were detected by western blot. (E) The interactions between NEK7 and WT or mutant NLRP3 in HEK-293T cells treated with or without 149-01 (3 $\mu \mathrm{M})$ were evaluated by IP and western blot. (F,G) NIrp3 ${ }^{-/-}$BMDMs reconstituted with mouse WT or mutant NLRP3 were first primed with LPS, then treated with or without 149-01 (600 nM) for $30 \mathrm{~min}$, lastly stimulated with nigericin. (F) IL-1 $\beta$ releases in supernatants were detected by ELISA. (G) Active IL-1 $\beta$ and p20 in supernatants and NLRP3, pro-IL-1 $\beta$, pro-caspase- 1 and $\beta$-actin in cell lysates were measured by western blot. Data are obtained from three independent experiments, each with two biological replicates and are expressed as mean \pm s. e.m $(n=6)(\mathbf{F})$, or are representative of three independent experiments (A-E,G). One-way ANOVA was applied to calculate statistical significance: ${ }^{\star * *} p<.001$, NS, not significant.

improved their clinical behaviors (Figure 7A). Consistent with this phenotype, in mice 14 days after EAE induction, HE staining revealed that 149-01 treatment ameliorated inflammatory cell infiltration in the spinal cord and LFB staining showed that 14901 treatment reduced spinal cord demyelination (Figure 7B). In addition, the proportions and absolute numbers of infiltrated $\mathrm{T}$ cells $\left(\mathrm{CD}^{+}\right.$and $\left.\mathrm{CD} 4^{+}\right)$, total lymphocytes $\left(\mathrm{CD} 11 \mathrm{~b}^{-} \mathrm{CD} 45^{\mathrm{hi}}\right)$, activated resident microglial cells and myeloid cells $\left(\mathrm{CD} 11 \mathrm{~b}^{+}\right.$ $\mathrm{CD} 45^{\mathrm{hi}}$ ) gated on $\mathrm{CD} 45^{\mathrm{hi}}$ cell populations in the CNS were markedly reduced in 149-01-treated mice (Figures 7C,D). Further investigation of inflammation in EAE mice showed that 149-01 treatment significantly inhibited the expression of inflammatory cytokines in the CNS, including IL-1 $\beta$, IL- 6 and TNF- $\alpha$ (Figure 7E). Furthermore, 149-01 treatment significantly reduced protein levels for IL-1 $\beta$, caspase- 1 and NLRP3 in the spinal cords of EAE mice (Figures 7F,G). Collectively, these findings suggest that the disease progression of EAE can be attenuated effectively by $149-01$ administration. To confirm that 149-01 mitigate EAE severity through suppression of NLRP3 inflammasome, we induced EAE in NLRP3 knockout mice. We found that treatment of $N L R P 3^{-/-}$mice with 149-01 did not affect the clinical disease scores of EAE (Figure $7 \mathbf{H}$ ) or the proportions and numbers of infiltrated immune cells in the CNS (Figures 7I,J). We also evaluated the safety profile of 149-01 in naive mice. Mice were intraperitoneally injected with $5 \mathrm{mg} / \mathrm{kg}$ 149-01 or vehicle control once a day for 4 weeks, and the results showed that 149-01 treatment did not affect the metabolic parameters and serum chemistry of naive mice (Supplementary Figure S7). Taken together, our results demonstrate that $149-01$ is active in vivo and can mitigate the progression of inflammatory diseases by targeting NLRP3 inflammasome.

\section{DISCUSSION}

In this study, we identify compound 149-01, an RRx-001 analogue, as a potent, specific and covalent NLRP3 inhibitor, which can effectively inhibit the NLRP3 inflammasome activation both in vitro and in vivo. Our study suggests that 149-01 may be a useful small molecule tool to investigate the mechanism of NLRP3 inflammasome activation and a potential lead for 
A

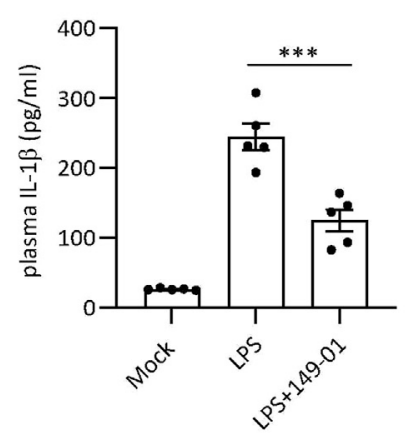

C

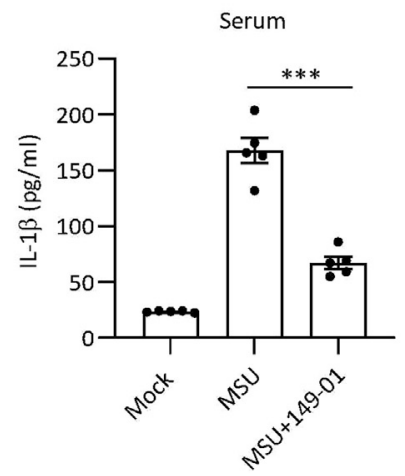

B

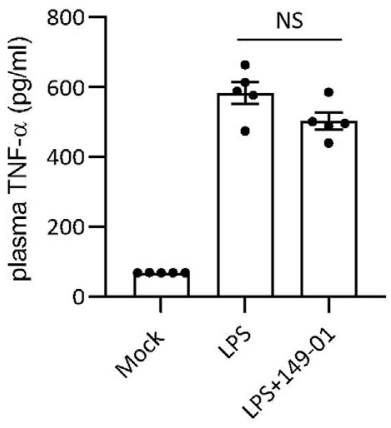

D

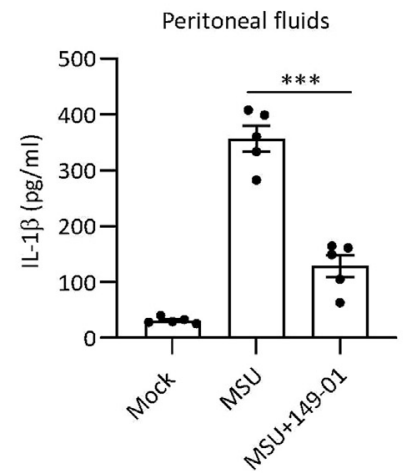

E

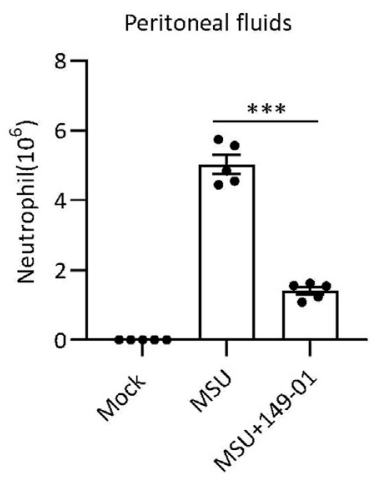

FIGURE 6 | 149-01 alleviates LPS-induced systemic inflammation and MSU-induced peritonitis in mice. (A,B) Serum IL-1 $\beta$ (A) and TNF- $\alpha$ (B) levels in mice intraperitoneally injected with 149-01 (5 mg/ $\mathrm{kg}$ ) or vehicle before challenge with LPS $(20 \mathrm{mg} / \mathrm{kg})$ were measured by ELISA. (C-E) Mice were intraperitoneally injected with 149-01 (5 mg/kg) or vehicle before challenge with MSU (1 mg/mouse). IL-1 $\beta$ in the serum (C) or peritoneal cavity (D) were measured by ELISA. (E) Neutrophil numbers in the peritoneal cavity were determined by FACS. Data are expressed as mean \pm s. e.m $(n=5)$. One-way ANOVA was applied to calculate statistical significance: ${ }^{* \star *} p<.001$, NS, not significant.

developing therapeutic agent for NLRP3-driven inflammatory diseases.

Our results showed that the IC50 (half-maximal inhibitory concentration) value of $149-01$ inhibiting IL- $1 \beta$ release is $182.8 \mathrm{nM}$, indicating that its inhibitory activity is stronger than most of the reported NLRP3 inhibitors, and 149-01 exhibits comparable inhibitory activity against NLRP3 inflammasome as RRx-001 (Chen et al., 2021). At the doses of 200-600 nM, 149-01 dose-dependently suppressed canonical and non-canonical NLRP3 activation in mouse cells and canonical and alternative NLRP3 activation in human cells. Meanwhile, 149-01 had no effect on the activation of AIM2, NLRC4, or Pyrin inflammasome, nor did it affect the priming phase of NLRP3 inflammasome at this concentration. These results demonstrate that 149-01 displays potent, broad and specific inhibitory effects on NLRP3 activation. We also observed that 149-01 treatment before LPS stimulation significantly inhibited LPS-induced proIL- $1 \beta$ and IL- 6 production at higher doses of $1-4 \mu \mathrm{M}$, and the concentration required to inhibit IL- 6 release is $\sim 9$ times higher than that required to inhibit IL- $1 \beta$ release, suggesting that 149-01 may have better therapeutic activity in NLRP3-related inflammatory disorders than in other inflammatory disorders.
However, the specific mechanism by which high doses of 149-01 inhibits LPS-induced pro-IL-1 $\beta$ and IL-6 production and its possible effects remain unclear. One possibility is that high doses of 149-01 also affect the function of other proteins, and high doses of 149-01 affect pro-IL-1 $\beta$ and IL- 6 production by targeting other proteins.

Mechanistically, our data indicated that 149-01 does not block NLRP3 inflammasome activation by affecting upstream events, including $\mathrm{K}^{+}$efflux, $\mathrm{Cl}^{-}$efflux and mitochondrial damage (Zhou et al., 2011; Munoz-Planillo et al., 2013; Gurung et al., 2015; Tang et al., 2017), suggesting that 14901 may directly affect the assembly of NLRP3 inflammasome complex. Indeed, 149-01 blocked the interaction between NEK7 and NLRP3 by irreversibly targeting cysteine 409 of NLRP3, ultimately inhibiting the NLRP3 inflammasome assembly and activation. However, according to the determined cryo-electron microscopy structure of human NLRP3-NEK7 complex (Sharif et al., 2019), we found that cysteine 409 is not at the interface of NLRP3-NEK7 interaction. We speculate that 149-01 binding to the C409 site of NLRP3 may cause a conformational change of NLRP3, resulting in the blockage of the NLRP3-NEK7 interaction, but 
A

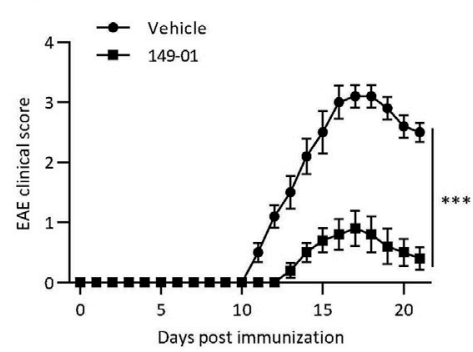

B
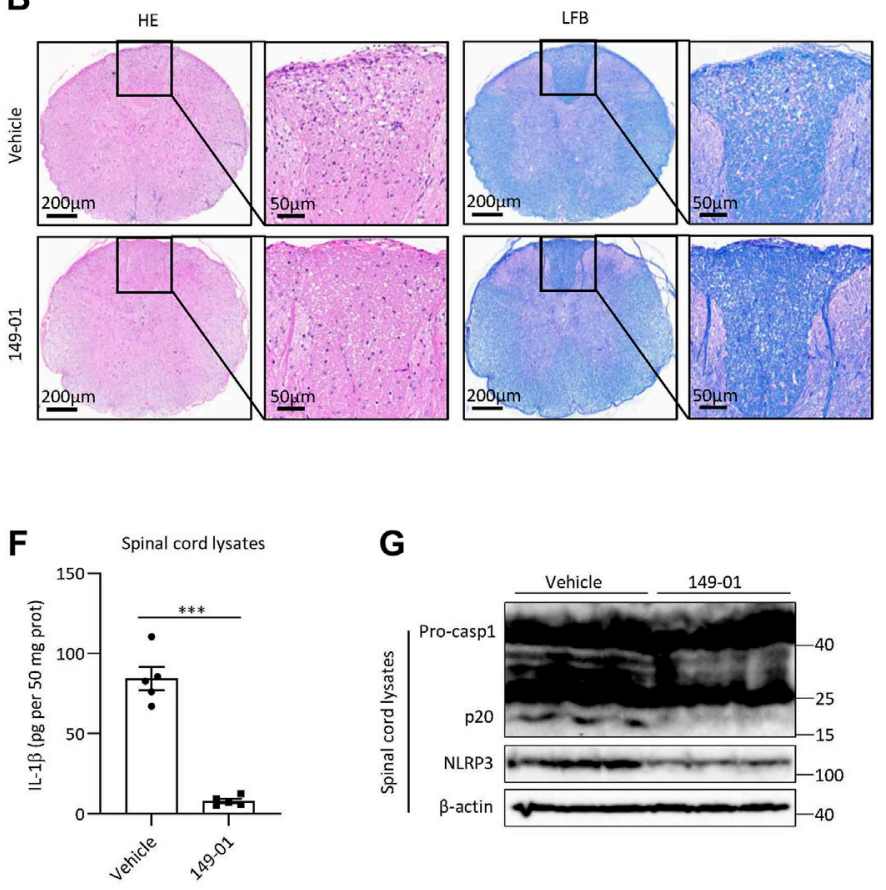

G

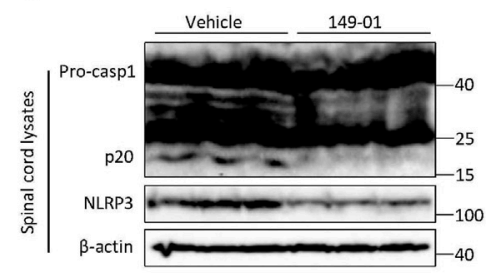

C
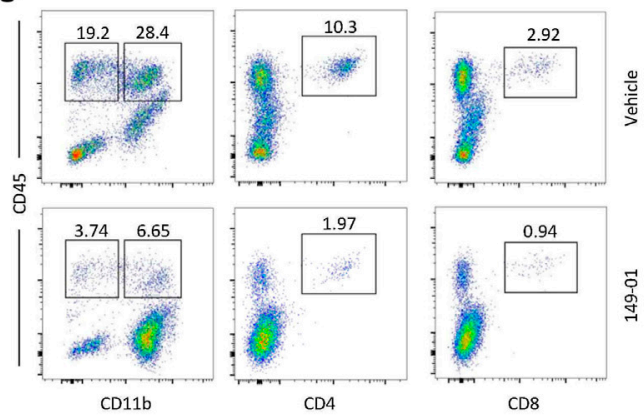

D

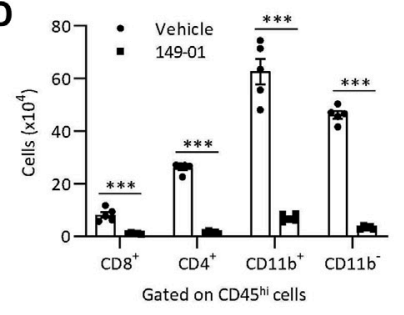

E

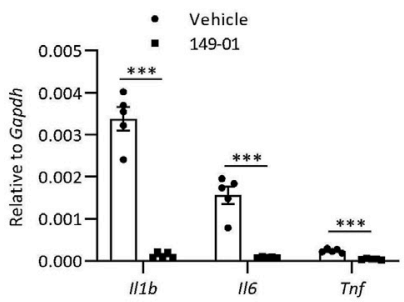

H

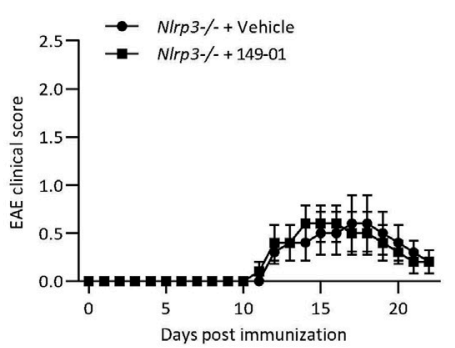

J

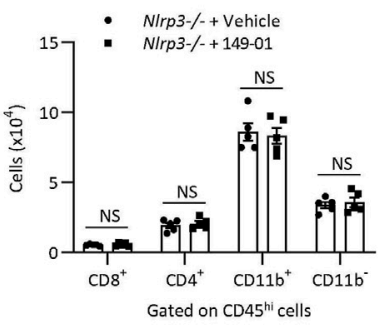

FIGURE 7| 149-01 attenuates the progression of EAE via inhibition of NLRP3 inflammasome. (A-G) Mice were intraperitoneally injected with 149-01 (5 mg/kg) or vehicle on the day of EAE induction and every 2 days thereafter. (A) EAE clinical scores. (B) Representative spinal cord sections were stained with H\&E or Luxol fast blue (LFB) to visualize inflammatory cell infiltration or demyelination at day 14 after EAE induction. (C,D) The percentages of representative plots (C) and total numbers (D) of infiltrated $\mathrm{CD}^{+}, \mathrm{CD}^{+}, \mathrm{CD} 11 \mathrm{~b}^{+}$and $\mathrm{CD} 11 \mathrm{~b}^{-}$cells gated on CD45 ${ }^{\text {hi }}$ cell populations in the CNS were determined by FACS at day 14 after EAE induction. (E) The expression of indicated inflammatory cytokines in the CNS were detected by qPCR at day 14 after EAE induction. (F,G) The protein levels of IL-1 $\beta$, caspase- 1 and NLRP3 in the spinal cords were detected by ELISA (F) or western blot (G) at day 14 after EAE induction. (H-J) NIrp3 ${ }^{-/-}$mice were intraperitoneally injected with $149-01$ 
FIGURE 7 | (5 mg/kg) or vehicle on the day of EAE induction and every 2 days thereafter. (H) EAE clinical scores. (I,J) The percentages of representative plots (I) and total numbers $(\mathbf{J})$ of infiltrated $\mathrm{CD} 8^{+}, \mathrm{CD} 4^{+}, \mathrm{CD} 11 \mathrm{~b}^{+}$and $\mathrm{CD} 11 \mathrm{~b}^{-}$cells gated on CD45 ${ }^{\text {hi }}$ cell populations in the CNS were determined by FACS at day 14 after EAE induction. Data are expressed as mean \pm s. e.m $(n=5)(\mathbf{A}, \mathbf{D}-\mathbf{F}, \mathbf{H}, \mathbf{J})$ or are representative of five mice (B,C,G,I). Unpaired Student's t-tests were applied to calculate statistical significance: ${ }^{* \star} p<.001$, NS, not significant.

how 149-01 binds to the cysteine 409 of NLRP3 and how this combination changes the conformation of NLRP3 remain to be further studied.

Our data revealed that 149-01 effectively alleviate LPS-induced systemic inflammation, MSU-induced peritonitis and EAE at a dose of $5 \mathrm{mg} / \mathrm{kg}$ and its therapeutic effect is dependent on NLRP3. In previous study, the dosage of $10 \mathrm{mg} / \mathrm{kg} \mathrm{RRx}-001$, was used in the treatment of multiple NLRP3-dependent disease models (Chen et al., 2021), indicating that 149-01 may possess in vivo therapeutic efficacy comparable to or better than RRx-001. Notably, in EAE mice model, 149-01 treatment significantly inhibited the expression of inflammatory cytokines in the CNS such as IL-6 and TNF- $\alpha$ in contrast to in vitro results. We suggested that the reduced inflammatory cytokines in the CNS may result from 1) 149-01 treatment alleviated the infiltration of inflammatory cell that produce these inflammatory cytokines; and 2) 149-01 treatment inhibited the expression of IL-1 $\beta$ which could mediate these inflammatory cytokines production via IL-1 receptors (Mori et al., 2011; Dinarello, 2018). Since RRx-001 has been shown to have a high safety profile in clinical trials (Reid et al., 2015; Oronsky et al., 2017; Oronsky et al., 2019), its analogue, 149-01, may have a higher potential to enter clinical trials.

Compared with RRx-001, the structure of 149-01 does not contain high-energy nitro functional groups, which avoids the side effects of NO production on the treatment of NLRP3-related diseases. In addition, 149-01 also avoids potential processing problems, for example, 1) the gem-dinitroazetidine can decompose rapidly, resulting in decreased yields; and 2) the gem-dinitroazetidine is sensitive to heat, impact, friction and electrostatic discharge, causing potential safety hazards (Straessler et al., 2012).

It has been proposed that inhibitors directly targeting NLRP3 have certain advantages over biologic agents targeting IL- $1 \beta$ in the treatment of NLRP3-related inflammatory disorders (Jiang et al., 2017), but there are still no clinically available therapeutic drugs. Although at least some small molecules have entered clinical trials, their clinical efficacy and safety remain to be further confirmed (Mullard, 2019; Kluck et al., 2020).

Considering the potent and specific inhibitory effect of 149-01 on NLRP3 inflammasome both in vitro and in vivo, and the high safety of RRx-001 in clinical trials (Reid et al., 2015; Oronsky et al., 2017; Oronsky et al., 2019), we consider that 149-01, as an analogue of $\mathrm{RRx}-001$, has promising therapeutic potential for NLRP3-related diseases. However, as mentioned above, there are some concerns remain to be further investigated, which may limit the application of 149-01 as a NLRP3 inhibitor.

\section{DATA AVAILABILITY STATEMENT}

The original contributions presented in the study are included in the article/Supplementary Materials, further inquiries can be directed to the corresponding authors.

\section{ETHICS STATEMENT}

The studies involving human participants were reviewed and approved by The University of Science and Technology of China. The patients/participants provided their written informed consent to participate in this study. The animal study was reviewed and approved by The University of Science and Technology of China.

\section{AUTHOR CONTRIBUTIONS}

HL, MY, CL, and BL performed the experiments of this work. XD, $\mathrm{HH}$, and RZ designed the research. HL and RZ wrote the manuscript. $\mathrm{HH}$ and $\mathrm{RZ}$ supervised the project.

\section{FUNDING}

This research was supported by the National Key research and development program of China (grant numbers 2018YFA0507403 and 2019YFA0508503), the Strategic Priority Research Program of the Chinese Academy of Sciences (grant number XDB29030102), the National Natural Science Foundation of China (grant numbers 82003765, 81821001, 31770991, and 82130107), the Fundamental Research Funds for the Central Universities, the University Synergy Innovation Program of Anhui Province (GXXT-2019-026) and the Natural Science Foundation of Anhui Province (1908085QC99).

\section{SUPPLEMENTARY MATERIAL}

The Supplementary Material for this article can be found online at: https://www.frontiersin.org/articles/10.3389/fphar.2022.822833/ full\#supplementary-material 


\section{REFERENCES}

Bauer, C., Duewell, P., Mayer, C., Lehr, H. A., Fitzgerald, K. A., Dauer, M., et al. (2010). Colitis Induced in Mice with Dextran Sulfate Sodium (DSS) Is Mediated by the NLRP3 Inflammasome. Gut 59 (9), 1192-1199. doi:10. 1136/gut.2009.197822

Broz, P., and Dixit, V. M. (2016). Inflammasomes: Mechanism of Assembly, Regulation and Signalling. Nat. Rev. Immunol. 16 (7), 407-420. doi:10. 1038/nri.2016.58

Chen, Y., He, H., Lin, B., Chen, Y., Deng, X., Jiang, W., et al. (2021). RRx-001 Ameliorates Inflammatory Diseases by Acting as a Potent Covalent NLRP3 Inhibitor. Cell Mol. Immunol. 18 (6), 1425-1436. doi:10.1038/s41423-02100683-y

Coll, R. C., Robertson, A. A., Chae, J. J., Higgins, S. C., Muñoz-Planillo, R., Inserra, M. C., et al. (2015). A Small-Molecule Inhibitor of the NLRP3 Inflammasome for the Treatment of Inflammatory Diseases. Nat. Med. 21 (3), 248-255. doi:10.1038/nm.3806

Coll, R. C., Hill, J. R., Day, C. J., Zamoshnikova, A., Boucher, D., Massey, N. L., et al. (2019). MCC950 Directly Targets the NLRP3 ATP-Hydrolysis Motif for Inflammasome Inhibition. Nat. Chem. Biol. 15 (6), 556-559. doi:10. 1038/s41589-019-0277-7

Daniels, M. J., Rivers-Auty, J., Schilling, T., Spencer, N. G., Watremez, W., Fasolino, V., et al. (2016). Fenamate NSAIDs Inhibit the NLRP3 Inflammasome and Protect against Alzheimer's Disease in Rodent Models. Nat. Commun. 7, 12504. doi:10.1038/ncomms12504

Davis, B. K., Wen, H., and Ting, J. P. (2011). The Inflammasome NLRs in Immunity, Inflammation, and Associated Diseases. Annu. Rev. Immunol. 29, 707-735. doi:10.1146/annurev-immunol-031210-101405

Dick, M. S., Sborgi, L., Rühl, S., Hiller, S., and Broz, P. (2016). ASC Filament Formation Serves as a Signal Amplification Mechanism for Inflammasomes. Nat. Commun. 7, 11929. doi:10.1038/ncomms11929

Dinarello, C. A., and van der Meer, J. W. (2013). Treating Inflammation by Blocking Interleukin-1 in Humans. Semin. Immunol. 25 (6), 469-484. doi:10.1016/j.smim.2013.10.008

Dinarello, C. A., Simon, A., and van der Meer, J. W. (2012). Treating Inflammation by Blocking Interleukin-1 in a Broad Spectrum of Diseases. Nat. Rev. Drug Discov. 11 (8), 633-652. doi:10.1038/nrd3800

Dinarello, C. A. (2018). Overview of the IL-1 Family in Innate Inflammation and Acquired Immunity. Immunol. Rev. 281 (1), 8-27. doi:10.1111/imr. 12621

Duewell, P., Kono, H., Rayner, K. J., Sirois, C. M., Vladimer, G., Bauernfeind, F. G., et al. (2010). NLRP3 Inflammasomes Are Required for Atherogenesis and Activated by Cholesterol Crystals. Nature 464 (7293), 1357-1361. doi:10.1038/nature08938

Duncan, J. A., Bergstralh, D. T., Wang, Y., Willingham, S. B., Ye, Z., Zimmermann, A. G., et al. (2007). Cryopyrin/NALP3 Binds ATP/dATP, Is an ATPase, and Requires ATP Binding to Mediate Inflammatory Signaling. Proc. Natl. Acad. Sci. U S A. 104 (19), 8041-8046. doi:10. 1073/pnas.0611496104

Fens, M. H. A. M., Larkin, S. K., Morris, C. R., Fitch, B., Scicinski, J., Oronsky, B., et al. (2011). NO or No NO, Increased Reduction of Nitrite to Nitric Oxide by Modified Red Blood Cells. Blood 118 (21), 2125. doi:10.1182/blood.V118.21. 2125.2125

Gris, D., Ye, Z., Iocca, H. A., Wen, H., Craven, R. R., Gris, P., et al. (2010). NLRP3 Plays a Critical Role in the Development of Experimental Autoimmune Encephalomyelitis by Mediating Th1 and Th17 Responses. J. Immunol. 185 (2), 974-981. doi:10.4049/jimmunol.0904145

Gross, C. J., Mishra, R., Schneider, K. S., Médard, G., Wettmarshausen, J., Dittlein, D. C., et al. (2016). K+ Efflux-independent NLRP3 Inflammasome Activation by Small Molecules Targeting Mitochondria. Immunity 45 (4), 761-773. doi:10.1016/j.immuni.2016.08.010

Guo, H., Callaway, J. B., and Ting, J. P. (2015). Inflammasomes: Mechanism of Action, Role in Disease, and Therapeutics. Nat. Med. 21 (7), 677-687. doi: $10.1038 / \mathrm{nm} .3893$

Gurung, P., Lukens, J. R., and Kanneganti, T. D. (2015). Mitochondria: Diversity in the Regulation of the NLRP3 Inflammasome. Trends Mol. Med. 21 (3), 193-201. doi:10.1016/j.molmed.2014.11.008
Haq, T., Richards, M. W., Burgess, S. G., Gallego, P., Yeoh, S., O'Regan, L., et al. (2015). Mechanistic Basis of Nek7 Activation through Nek9 Binding and Induced Dimerization. Nat. Commun. 6, 8771. doi:10.1038/ncomms9771

He, Y., Franchi, L., and Núñez, G. (2013). TLR Agonists Stimulate Nlrp3dependent IL-1 $\beta$ Production Independently of the Purinergic P2X7 Receptor in Dendritic Cells and In Vivo. J. Immunol. 190 (1), 334-339. doi:10.4049/ jimmunol.1202737

He, Y., Zeng, M. Y., Yang, D., Motro, B., and Núñez, G. (2016). NEK7 Is an Essential Mediator of NLRP3 Activation Downstream of Potassium Efflux. Nature 530 (7590), 354-357. doi:10.1038/nature16959

He, H., Jiang, H., Chen, Y., Ye, J., Wang, A., Wang, C., et al. (2018). Oridonin Is a Covalent NLRP3 Inhibitor with strong Anti-inflammasome Activity. Nat. Commun. 9 (1), 2550. doi:10.1038/s41467-018-04947-6

Heneka, M. T., Kummer, M. P., Stutz, A., Delekate, A., Schwartz, S., Vieira-Saecker, A., et al. (2013). NLRP3 Is Activated in Alzheimer's Disease and Contributes to Pathology in APP/PS1 Mice. Nature 493 (7434), 674-678. doi:10.1038/nature11729

Huang, Y., Jiang, H., Chen, Y., Wang, X., Yang, Y., Tao, J., et al. (2018). Tranilast Directly Targets NLRP3 to Treat Inflammasome-Driven Diseases. EMBO Mol. Med. 10 (4), e8689. doi:10.15252/emmm.201708689

Jiang, H., He, H., Chen, Y., Huang, W., Cheng, J., Ye, J., et al. (2017). Identification of a Selective and Direct NLRP3 Inhibitor to Treat Inflammatory Disorders. J. Exp. Med. 214 (11), 3219-3238. doi:10.1084/jem.20171419

Kim, M. M., Parmar, H., Cao, Y., Pramanik, P., Schipper, M., Hayman, J., et al. (2016). Whole Brain Radiotherapy and RRx-001: Two Partial Responses in Radioresistant Melanoma Brain Metastases from a Phase I/II Clinical Trial: A TITE-CRM Phase I/II Clinical Trial. Transl. Oncol. 9 (2), 108-113. doi:10.1016/ j.tranon.2015.12.003

Klück, V., Jansen, T. L. T. A., Janssen, M., Comarniceanu, A., Efdé, M., Tengesdal, I. W., et al. (2020). Dapansutrile, an Oral Selective NLRP3 Inflammasome Inhibitor, for Treatment of Gout Flares: an Open-Label, Dose-Adaptive, Proof-Of-Concept, Phase 2a Trial. Lancet Rheumatol. 2 (5), e270-e280. doi:10.1016/s2665-9913(20)30065-5

Lalor, S. J., Dungan, L. S., Sutton, C. E., Basdeo, S. A., Fletcher, J. M., and Mills, K. H. (2011). Caspase-1-processed Cytokines IL-1beta and IL-18 Promote IL-17 Production by Gammadelta and CD4 T Cells that Mediate Autoimmunity. J. Immunol. 186 (10), 5738-5748. doi:10.4049/jimmunol.1003597

Lomenick, B., Hao, R., Jonai, N., Chin, R. M., Aghajan, M., Warburton, S., et al. (2009). Target Identification Using Drug Affinity Responsive Target Stability (DARTS). Proc. Natl. Acad. Sci. U S A. 106 (51), 21984-21989. doi:10.1073/ pnas. 0910040106

Lu, A., Magupalli, V. G., Ruan, J., Yin, Q., Atianand, M. K., Vos, M. R., et al. (2014). Unified Polymerization Mechanism for the Assembly of ASC-dependent Inflammasomes. Cell 156 (6), 1193-1206. doi:10.1016/j.cell.2014.02.008

Marchetti, C., Swartzwelter, B., Gamboni, F., Neff, C. P., Richter, K., Azam, T., et al. (2018). OLT1177, a $\beta$-sulfonyl Nitrile Compound, Safe in Humans, Inhibits the NLRP3 Inflammasome and Reverses the Metabolic Cost of Inflammation. Proc. Natl. Acad. Sci. U S A. 115 (7), E1530-E1539. doi:10.1073/pnas.1716095115

Martinon, F., Pétrilli, V., Mayor, A., Tardivel, A., and Tschopp, J. (2006). Goutassociated Uric Acid Crystals Activate the NALP3 Inflammasome. Nature 440 (7081), 237-241. doi:10.1038/nature04516

Masters, S. L., Dunne, A., Subramanian, S. L., Hull, R. L., Tannahill, G. M., Sharp, F. A., et al. (2010). Activation of the NLRP3 Inflammasome by Islet Amyloid Polypeptide Provides a Mechanism for Enhanced IL-1 $\beta$ in Type 2 Diabetes. Nat. Immunol. 11 (10), 897-904. doi:10.1038/ni.1935

Mori, T., Miyamoto, T., Yoshida, H., Asakawa, M., Kawasumi, M., Kobayashi, T., et al. (2011). IL-1 and TNF -initiated IL-6-STAT3 Pathway Is Critical in Mediating Inflammatory Cytokines and RANKL Expression in Inflammatory Arthritis. Int. Immunol. 23 (11), 701-712. doi:10.1093/ intimm/dxr077

Mullard, A. (2019). NLRP3 Inhibitors Stoke Anti-inflammatory Ambitions. Nat. Rev. Drug Discov. 18 (6), 405-407. doi:10.1038/d41573-019-00086-9

Muñoz-Planillo, R., Kuffa, P., Martínez-Colón, G., Smith, B. L., Rajendiran, T. M., and Núñez, G. (2013). $\mathrm{K}^{+}$Efflux Is the Common Trigger of NLRP3 Inflammasome Activation by Bacterial Toxins and Particulate Matter. Immunity 38 (6), 1142-1153. doi:10.1016/j.immuni.2013.05.016

Netea, M. G., van de Veerdonk, F. L., van der Meer, J. W., Dinarello, C. A., and Joosten, L. A. (2015). Inflammasome-independent Regulation of IL-1-family Cytokines. Аnпu. Rev. Immunol. 33, 49-77. doi:10.1146/annurev-immunol-032414-112306 
Ning, S., Bednarski, M., Oronsky, B., Scicinski, J., Saul, G., and Knox, S. J. (2012). Dinitroazetidines Are a Novel Class of Anticancer Agents and Hypoxia-Activated Radiation Sensitizers Developed from Highly Energetic Materials. Cancer Res. 72 (10), 2600-2608. doi:10.1158/0008-5472.CAN11-2303

Nowarski, R., Jackson, R., Gagliani, N., de Zoete, M. R., Palm, N. W., Bailis, W., et al. (2015). Epithelial IL-18 Equilibrium Controls Barrier Function in Colitis. Cell 163 (6), 1444-1456. doi:10.1016/j.cell.2015.10.072

Oronsky, B., Scicinski, J., Ning, S., Peehl, D., Oronsky, A., Cabrales, P., et al. (2016). Rockets, Radiosensitizers, and RRx-001: an Origin story Part I. Discov. Med. 21 (115), 173-180.

Oronsky, B., Paulmurugan, R., Foygel, K., Scicinski, J., Knox, S. J., Peehl, D., et al. (2017). RRx-001: a Systemically Non-toxic M2-To-M1 Macrophage Stimulating and Prosensitizing Agent in Phase II Clinical Trials. Expert Opin. Investig. Drugs 26 (1), 109-119. doi:10.1080/13543784.2017.1268600

Oronsky, B., Reid, T. R., Larson, C., Caroen, S., Quinn, M., Burbano, E., et al. (2019). REPLATINUM Phase III Randomized Study: RRx-001 + Platinum Doublet versus Platinum Doublet in Third-Line Small Cell Lung Cancer. Future Oncol. 15 (30), 3427-3433. doi:10.2217/fon-2019-0317

Reid, T., Oronsky, B., Scicinski, J., Scribner, C. L., Knox, S. J., Ning, S., et al. (2015). Safety and Activity of RRx-001 in Patients with Advanced Cancer: a First-In-Human, Open-Label, Dose-Escalation Phase 1 Study. Lancet Oncol. 16 (9), 1133-1142. doi:10.1016/S1470-2045(15)00089-3

Schroder, K., and Tschopp, J. (2010). The Inflammasomes. Cell 140 (6), 821-832. doi:10. 1016/j.cell.2010.01.040

Sharif, H., Wang, L., Wang, W. L., Magupalli, V. G., Andreeva, L., Qiao, Q., et al. (2019). Structural Mechanism for NEK7-Licensed Activation of NLRP3 Inflammasome. Nature 570 (7761), 338-343. doi:10.1038/s41586-019-1295-Z

Shi, J., Zhao, Y., Wang, K., Shi, X., Wang, Y., Huang, H., et al. (2015). Cleavage of GSDMD by Inflammatory Caspases Determines Pyroptotic Cell Death. Nature 526 (7575), 660-665. doi:10.1038/nature15514

Shi, H., Wang, Y., Li, X., Zhan, X., Tang, M., Fina, M., et al. (2016). NLRP3 Activation and Mitosis Are Mutually Exclusive Events Coordinated by NEK7, a New Inflammasome Component. Nat. Immunol. 17 (3), 250-258. doi:10.1038/ni.3333

Shi, Y., Lv, Q., Zheng, M., Sun, H., and Shi, F. (2021). NLRP3 Inflammasome Inhibitor INF39 Attenuated NLRP3 Assembly in Macrophages. Int. Immunopharmacol. 92, 107358. doi:10.1016/j.intimp.2020.107358

Straessler, N. A., Lesley, M. W., and Cannizzo, L. F. (2012). Development of a Safe and Efficient Two-step Synthesis for Preparing 1-Bromoacetyl-3,3Dinitroazetidine, a Novel Clinical Anticancer Candidate. Org. Process. Res. Dev. 16, 512-517. doi:10.1021/op2003216

Sutton, C., Brereton, C., Keogh, B., Mills, K. H., and Lavelle, E. C. (2006). A Crucial Role for Interleukin (IL)-1 in the Induction of IL-17-producing T Cells that Mediate Autoimmune Encephalomyelitis. J. Exp. Med. 203 (7), 1685-1691. doi:10.1084/jem.20060285
Swanson, K. V., Deng, M., and Ting, J. P. (2019). The NLRP3 Inflammasome: Molecular Activation and Regulation to Therapeutics. Nat. Rev. Immunol. 19 (8), 477-489. doi:10.1038/s41577-019-0165-0

Tang, T., Lang, X., Xu, C., Wang, X., Gong, T., Yang, Y., et al. (2017). CLICsDependent Chloride Efflux Is an Essential and Proximal Upstream Event for NLRP3 Inflammasome Activation. Nat. Commun. 8 (1), 202. doi:10. 1038/s41467-017-00227-x

van der Vliet, A., Eiserich, J. P., and Cross, C. E. (2000). Nitric Oxide: a Proinflammatory Mediator in Lung Disease? Respir. Res. 1 (2), 67-72. doi:10. $1186 / \mathrm{rr} 14$

Wen, H., Ting, J. P., and O'Neill, L. A. (2012). A Role for the NLRP3 Inflammasome in Metabolic Diseases-Ddid Warburg Miss Inflammation? Nat. Immunol. 13 (4), 352-357. doi:10.1038/ni.2228

Xu, H., Yang, J., Gao, W., Li, L., Li, P., Zhang, L., et al. (2014). Innate Immune Sensing of Bacterial Modifications of Rho GTPases by the Pyrin Inflammasome. Nature 513 (7517), 237-241. doi:10.1038/nature13449

Yu, W., Wang, Z., Zhang, K., Chi, Z., Xu, T., Jiang, D., et al. (2019). OneCarbon Metabolism Supports S-Adenosylmethionine and Histone Methylation to Drive Inflammatory Macrophages. Mol. Cel 75 (6), 1147. doi:10.1016/j.molcel.2019.06.039

Zhang, X., Xu, A., Lv, J., Zhang, Q., Ran, Y., Wei, C., et al. (2020). Development of Small Molecule Inhibitors Targeting NLRP3 Inflammasome Pathway for Inflammatory Diseases. Eur. J. Med. Chem. 185, 111822. doi:10.1016/j.ejmech.2019.111822

Zhou, R., Yazdi, A. S., Menu, P., and Tschopp, J. (2011). A Role for Mitochondria in NLRP3 Inflammasome Activation. Nature 469 (7329), 221-225. doi:10.1038/nature09663

Conflict of Interest: The authors declare that the research was conducted in the absence of any commercial or financial relationships that could be construed as a potential conflict of interest.

Publisher's Note: All claims expressed in this article are solely those of the authors and do not necessarily represent those of their affiliated organizations, or those of the publisher, the editors and the reviewers. Any product that may be evaluated in this article, or claim that may be made by its manufacturer, is not guaranteed or endorsed by the publisher.

Copyright $\odot 2022$ Lin, Yang, Li, Lin, Deng, He and Zhou. This is an open-access article distributed under the terms of the Creative Commons Attribution License (CC BY). The use, distribution or reproduction in other forums is permitted, provided the original author(s) and the copyright owner(s) are credited and that the original publication in this journal is cited, in accordance with accepted academic practice. No use, distribution or reproduction is permitted which does not comply with these terms. 